Title: Analytical Interfacial Layer Model for the Capacitance and Electrokinetics of Charged Aqueous Interfaces

Author(s): Uematsu, Y., Netz, R. R., \& Bonthuis, D. J.

Document type: Postprint

Terms of Use: $\quad$ Copyright applies. A non-exclusive, non-transferable and limited right to use is granted. This document is intended solely for personal, non-commercial use.

Citation:

This document is the Accepted Manuscript version of a Published Work that appeared in final form in Langmuir, copyright $\odot$ American Chemical Society after peer review and technical editing by the publisher. To access the final edited and published work see https://doi.org/10.1021/acs.langmuir.7b04171.

Uematsu, Y., Netz, R. R., \& Bonthuis, D. J. (2018). Analytical Interfacial Layer Model for the Capacitance and Electrokinetics of Charged Aqueous Interfaces. Langmuir, 34(31), 90979113. https://doi.org/10.1021/acs.langmuir. 7 b04171 


\title{
Analytical interfacial layer model for the capacitance and electrokinetics of charged aqueous interfaces
}

\author{
Yuki Uematsu, ${ }^{*,+, \ddagger}$ Roland R. Netz, ${ }^{*, \ddagger}$ and Douwe Jan Bonthuis ${ }^{\ddagger}$ \\ $\dagger$ Department of Chemistry, Kyushu University, Fukuoka 819-0395, Japan \\ $\ddagger$ Fachbereich Physik, Freie Universität Berlin, 14195 Berlin, Germany \\ E-mail: uematsu@chem.kyushu-univ.jp; rnetz@physik.fu-berlin.de
}

\begin{abstract}
We construct an analytical model to account for the influence of the subnanometerwide interfacial layer on the differential capacitance and the electro-osmotic mobility of solid-electrolyte interfaces. The interfacial layer is incorporated into the PoissonBoltzmann and Stokes equations using a box model for the dielectric properties, the viscosity and the ionic potential of mean force. We calculate the differential capacitance and the electro-osmotic mobility as a function of the surface charge density and the salt concentration, both with and without steric interactions between the ions. We compare the results from our theoretical model with experimental data on a variety of systems (graphite and metallic silver for capacitance and titanium oxide and silver iodide for electro-osmotic data). The differential capacitance of silver as a function of salinity and surface charge density is well reproduced by our theory, using either the width of the interfacial layer or the ionic potential of mean force as the only fitting parameter. The differential capacitance of graphite, however, needs an additional carbon capacitance to explain the experimental data. Our theory yields a power-law dependence of
\end{abstract}


the electro-osmotic mobility on the surface charge density for high surface charges, reproducing the experimental data both using the interfacial parameters extracted from molecular dynamics simulations, as well as using fitted interfacial parameters. Finally, we examine different types of hydrodynamic boundary conditions for the power-law behavior of the electro-osmotic mobility, showing that a finite-viscosity layer explains the experimental data better than the usual hydrodynamic slip boundary condition. Our analytical model thus allows us to extract the properties of the subnanometer-wide interfacial layer by fitting to macroscopic experimental data.

\section{Introduction}

The electric double layer, formed by ions which are electrostatically attracted or otherwise adsorbed at interfaces, plays an essential role in electrochemistry, colloidal physics, and interfacial science. ${ }^{1-3}$ In particular, a charged surface and its counterions constitute a nanometer-scale capacitor, the capacitance of which depends sensitively on the properties of the interfacial layer. When a lateral electric field or pressure gradient is applied, the double layer gives rise to electrokinetic transport phenomena. Both the interfacial capacitance and the electrokinetic transport are of notable interest in many industrial and biological processes. ${ }^{1-3}$ The traditional model describing both the double layer capacitance and electrokinetics consists of the Poisson-Boltzmann (PB) and hydrodynamic (Stokes) equations ${ }^{1-3}$ and is based on point charges in a homogeneous viscous fluid. However, the classical theory cannot consistently reproduce some experimental observations, such as the significantly reduced differential capacitance, ${ }^{4,5}$ the saturation of the electro-osmotic flow with rising surface charge density, ${ }^{6,7}$ ion-specific effects, ${ }^{8,9}$ overscreening in ionic liquids ${ }^{10}$, and interactions between surfaces in water when they are close together. ${ }^{11,12}$

Seeking to capture these effects, many modifications of the PB equation have been published over the past decades. It has been recognized early in the 20th century that the dielectric environment of water is not spatially homogeneous, and several modified PB equa- 
tions incorporate the dielectric decrement due to the presence of ions. ${ }^{13,14}$ Attempts to also include water-water and water-surface interactions on the dielectric environment has led to the development of the dipolar PB equation. ${ }^{15}$ Another key approximation in the tradition model, the point-charge nature of the ions, has been circumvented through the introduction of a finite ion size. ${ }^{16-19}$ Other models for the ion's steric effects have also been suggested, such as one based on the virial expansion of the equation of state of a hard-sphere liquid. ${ }^{20,21}$ The differences between these different steric interaction models for the predicted electrophoretic mobility of spherical particles with fixed charge density have been found to be minor. ${ }^{22}$ As an alternative to these adjustments, the PB equation has been modified by incorporating a potential of mean force in addition to the electrostatic potential. ${ }^{9,23,24}$ For example, the potential of mean force between surfaces and hydrated ions has been extracted from classical molecular dynamics (MD) simulations, ${ }^{24,25}$ taking into account the combined effects of ion-specific interactions, dielectric profile, steric interactions and image charge effects.

More recently, the interfacial structure of water itself has been studied using MD simulations, ${ }^{26-30}$ revealing that the dielectric constant of pure water is highly non-uniform near the interface, oscillating and reversing sign repeatedly. The same behavior has been observed for water confined between polar soft surfaces. ${ }^{31}$ This inhomogeneous dielectric profile reduces the differential capacitance because the spatial average of the inverse dielectric profile near the surface is higher than the inverse of the bulk dielectric constant at both hydrophilic and hydrophobic surfaces. Apart from the dielectric profile, the viscosity near the interface is also non-uniform, and strongly depends on the affinity between the surface and the solvent. Results obtained for uncharged surfaces show an increased viscosity at hydrophilic surfaces, ${ }^{26,27}$ which can be modeled by a stagnant layer of fluid. At hydrophobic surfaces, however, a water-depleted layer is observed, modeled by a layer of decreased viscosity or by a finite slip length. ${ }^{26,27}$ The slip length depends sensitively on the presence of a surface charge, ${ }^{32}$ but the relation between the interfacial structure and charge density, surface slip and the interfacial viscosity is not clear yet. The PB and Stokes equations including the 
effects of these inhomogeneous profiles for the dielectric constant and the viscosity, as well as ion-specific interactions, have been solved numerically, and the resultant electrokinetic flow (expressed as an effective surface charge density) as a function of the titrated surface charge density is in excellent agreement with experimental data without using viscoelectric effects. $^{27,30}$ The differential capacitance at the point of zero charge (PZC) has also been analyzed using this framework for carbon surfaces, ${ }^{28,29}$ but an analysis for metals is still lacking.

With these modified profiles, the PB equation cannot be solved analytically, but an analytical solution exists if the profiles are approximated by step functions. In contrast to the numerical solutions, analytical expressions for the electrokinetic flow and the interfacial capacitance allow the effective parameters characterizing the double layer to be deduced directly from experimental data by fitting. The primary challenge now is to develop an analytically tractable model capable of explaining the multitude of experimental observations simultaneously. In this work, we focus on a simultaneous description of the differential capacitance and the electrokinetic mobility.

The differential capacitance, which characterizes how much charge is stored at an interface between a solid and an electrolyte, has been measured for many different combinations of conducting materials and aqueous electrolytes, ${ }^{5,33,34}$ typically by measuring the current in response to an applied alternating current $(\mathrm{AC})$ voltage, see figure 1a. It depends on the applied potential in a nonlinear fashion, as well as on the type of ions in the electrolyte. ${ }^{33,34}$ For the capacitance of metal-electrolyte interfaces at the PZC, the standard PB equation predicts higher values than those measured experimentally in the case of high salinity. At low salinity, however, the experimental capacitance of metal-electrolyte interfaces mostly agrees with, and sometimes exceeds, the prediction of the standard PB equation. In addition to the standard PB equation, the phenomenological framework available to describe this data consists of an excess interfacial capacitance and a finite roughness of the metallic surfaces, both of which are extracted from the experimental data via Parsons-Zobel plots. ${ }^{33-35}$ For 
carbonic materials like graphites ${ }^{36,37}$ and graphenes, ${ }^{38}$ the experimental differential capacitance is generally much lower than both the capacitance of metal-electrolyte interfaces and the standard PB prediction. To address these discrepancies, Stern considered the effects of the dielectric properties of the interfacial layer, as well as ion adsorption at large potentials $^{39}$ in his phenomenological model of the interfacial capacitance. ${ }^{4}$ This Stern layer effect is also important for electrification of colloidal surfaces, ${ }^{40}$ electro-osmosis ${ }^{41}$ and electrophoresis. ${ }^{42}$ At large potentials, steric interactions between the ions have been identified as the cause of the experimentally observed maximum in the capacitance. ${ }^{16,17,19,22,43-50}$ Despite the long-standing interest in the interfacial capacitance from both theoretical and applied perspectives, however, a unified model for this phenomenon at varying potentials and salt concentrations is lacking so far.

The dynamics of the double layer determine the electrophoretic mobility of charged solutes in an electrolyte and the electro-osmotic flow at charged surfaces. The electrophoretic mobility of a spherical particle in solution has been intensely studied by the standard PB and Stokes equations, and the dependence of the electrophoretic mobility on the salt concentration, the particle radius and the surface potential is known. ${ }^{51}$ Specifically, in terms of the surface potential $\psi_{0}$, the dielectric constant $\varepsilon$ and the viscosity $\eta$, the mobility equals $\varepsilon \varepsilon_{0} \psi_{0} / \eta$ for arbitrary surface potential and large radius, known as the Helmholtz-Smoluchowski formula, ${ }^{52}$ and $(2 / 3) \varepsilon \varepsilon_{0} \psi_{0} / \eta$ for arbitrary surface potential and small radius. ${ }^{53,54}$ For intermediate radii, the electrophoretic mobility becomes nonlinear with respect to the surface potential, known as the relaxation effect or double layer polarization effect. ${ }^{51,55}$ In experiments, the electrokinetic surface charge density or the associated zeta potential are defined by the measured electro-osmotic or electrophoretic mobility via these standard equations, see figure 1b. 3,6,56 Generally, however, even if the double layer polarization effect is eliminated from the electrophoretic mobility, the electrokinetic surface charge density deviates from the bare surface charge density measured by titration. ${ }^{3,6,56-58}$ In fact, for strongly charged surfaces, the electrokinetic surface charge density is much smaller than the titrated surface 
charge density. One way to explain the difference between the electrokinetic and titrated surface charge densities is to assume that the no-slip boundary is not located at the solidliquid interface, but at a finite distance away from the interface. ${ }^{56,58}$ The layer between the solid surface and the no-slip boundary is called the stagnant layer and, according to the simplest model, has infinite viscosity. This way, however, the predicted saturated (high surface charge) zeta potential is much larger than the experimental one, and the predicted doublelayer conductivity contradicts experimental measurements. ${ }^{27,30}$ Another scenario to explain the difference between the electrokinetic and titrated surface charge densities is the viscoelectric effect, which refers to the enhancement of the local viscosity due to the double-layer electric field near the surface. ${ }^{56} \mathrm{~A}$ microscopic foundation of the viscoelectric effect is still lacking, however. Finally, for strong electric fields, the dielectric constant has been found to decrease as a function of applied field, ${ }^{59}$ which affects the electrostatic potential in the electric double layer. Even so, the latter can typically be neglected, since experimentally applied electric fields are small and because the effect of the interfacial structure dominates the effect of the applied field. ${ }^{29}$

In this paper, we explore the analytical solution of the PB equation with interfacial inhomogeneities beyond the previously reported power-law scaling of the electrokinetic mobility with surface charge. ${ }^{60}$ In particular, we study the differential capacitance within the same framework, both at the PZC and at a finite surface charge. We also examine the effects of steric interactions on the differential capacitance and on the electrokinetic power law, and study different descriptions of the finite-viscosity layer and the interfacial slip length. We fit our theory to the relevant experimental data sets which are available in the literature. Because to our knowledge no complete data set of electrokinetic flow, titrated surface charge density and interfacial capacitance for one surface exists, we are forced to use different data sets for the different aspects of our model. We hope that our analysis will encourage further experimental studies on the electric double layer.

This paper is organized as follows. In the second section, we describe the PB equation 
including inhomogeneous dielectric profiles and nonelectrostatic interactions between ions and surfaces, also accounting for the finite ion size. In the third section, we derive the exact solution of the $\mathrm{PB}$ equation with inhomogeneous dielectric profile and ion-specific interactions. In the fourth section we discuss the differential capacitance and in the fifth section we discuss the electrokinetic power law with different inhomogeneous viscosity profiles. We summarize the results in the last section.

(a) Capacitance

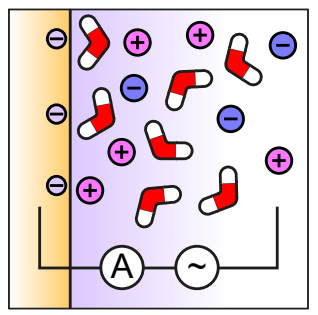

(b) Electrokinetics

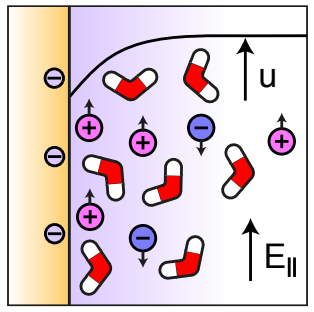

Figure 1: Illustration of the two types of experiments that we use as a reference in this work. (a) The interfacial capacitance is typically measured by measuring the electric current in response to an applied AC voltage. (b) The electrokinetic mobility is measured by measuring the velocity difference $u$ between the substrate and the fluid induced by an applied lateral electric field $E_{\|}$.

\section{The model}

\section{Extended Poisson-Boltzmann equations}

We consider a planar interface $(z=0)$ between an aqueous electrolyte solution $(z>0)$ and another liquid, solid or gas $(z<0)$. All quantities are laterally homogeneous, so the Poisson equation reads ${ }^{28,29}$

$$
\frac{d}{d z}\left[\varepsilon_{\perp}(z) \varepsilon_{0} \frac{d}{d z} \psi(z)\right]=-\rho(z)
$$

where $\varepsilon_{0}$ is the electric permittivity of vacuum and $\psi(z)$ is the local electrostatic potential. The local perpendicular dielectric constant $\varepsilon_{\perp}(z)$ can be derived from the general nonlocal inverse dielectric response function in the limit of a slowly varying displacement field. ${ }^{28}$ The 
ionic charge density $\rho(z)$ is given by

$$
\rho(z)=e\left(c^{+}(z)-c^{-}(z)\right)
$$

where $c^{ \pm}(z)$ is the local concentration of monovalent cations and anions and $e$ is the absolute value of the elementary charge. If we neglect the steric interactions between ions, the concentrations of cations and anions obey the Boltzmann distribution

$$
c^{ \pm}(z)=c_{0} \mathrm{e}^{\mp \Psi(z)-\mu_{0}^{ \pm}(z)}
$$

where $c_{0}$ is the bulk concentration of electrolyte, $\Psi(z)=e \psi(z) / k_{\mathrm{B}} T$ is the dimensionless potential with $k_{\mathrm{B}} T$ being the thermal energy, and $\mu_{0}^{ \pm}(z)$ is the nonelectrostatic interaction potential between the ions and the surface. If, instead, we include the steric interaction between ions, the ionic concentration can be written as ${ }^{16,19,43}$

$$
c^{ \pm}(z)=\frac{c_{0} \mathrm{e}^{\mp \Psi(z)-\mu_{0}^{ \pm}(z)}}{1+(\nu / 2)\left(\mathrm{e}^{-\Psi(z)-\mu_{0}^{+}(z)}+\mathrm{e}^{\Psi(z)-\mu_{0}^{-}(z)}-2\right)},
$$

where $\nu$ is the ionic packing parameter. The packing parameter has a relation with the effective ionic diameter $a$ via $\nu=\sqrt{2} c_{0} a^{3}(<1)$. This determines the maximum ion concentration $c^{ \pm}=\sqrt{2} / a^{3}$, which is the maximum density of close-packed (face-centered cubic or hexagonal close-packed) spheres of diameter $a$. In the following, models without steric effects are based on eq 3 and models with steric effects are based on eq 4. The boundary conditions for eq 1 are determined by the surface charge density $\sigma_{0}$ and a vanishing potential far from the surface,

$$
\left.\varepsilon_{\perp}(z) \varepsilon_{0} \frac{d \psi}{d z}\right|_{z=0}=-\sigma_{0} \quad \text { and }\left.\quad \psi(z)\right|_{z \rightarrow+\infty}=0 .
$$

At the surface, we do not consider any chemical reaction involving protons, ions and functional groups. Therefore, when studying the differential capacitance, the electrodes are assumed to be ideally polarizable and the surface charge density $\sigma_{0}$ is a given parameter which 
can be controlled by applying an electrostatic potential difference between the electrode and the bulk electrolyte. In the case of electro-osmosis, the surface charge density $\sigma_{0}$ can be controlled by adjusting the concentration of protons or potential-determining ions. In particular, when the proton or hydroxide concentration is well below the bulk salt concentration $\left(c_{0} \gg 10^{-\mathrm{pH}}\right.$ or $\left.c_{0} \gg 10^{\mathrm{pH}-14}\right)$, any $\sigma_{0}$ can be obtained by adjusting the $\mathrm{pH}$ and we may consider $\sigma_{0}$ as a given parameter without considering the chemical equilibrium. For analytical tractability, the non-uniform dielectric constant is modeled as a box profile $27-30,60,61$

$$
\varepsilon_{\perp}(z)= \begin{cases}\varepsilon_{\text {int }} & \text { for } 0<z<z_{\varepsilon} \\ \varepsilon & \text { for } z_{\varepsilon}<z\end{cases}
$$

where $\varepsilon$ is the dielectric constant of the bulk solution, $\varepsilon_{\text {int }}$ is the dielectric constant in the dielectric interfacial layer and $z_{\varepsilon}$ is the width of the dielectric interfacial layer. The additional non-electrostatic ion-surface interaction is modeled as

$$
\mu_{0}^{ \pm}(z)= \begin{cases}\alpha_{ \pm} & \text {for } 0<z<z_{\alpha}^{ \pm} \\ 0 & \text { for } z_{\alpha}^{ \pm}<z\end{cases}
$$

where $z_{\alpha}^{ \pm}$is the width of the adsorption layer and $\alpha_{ \pm}$is the ion-specific surface interaction parameter. If we consider the symmetric case $\alpha_{+}=\alpha_{-}$, we simply denote the ion-specific surface interaction parameter as $\alpha=\alpha_{+}=\alpha_{-}$. In general, $z_{\alpha}^{ \pm} \neq z_{\varepsilon}$, but in the following we consider only the case of $z_{\alpha}^{ \pm}=z_{\varepsilon}$ for analytical tractability. In the presence of steric effects, eq 1 yields

$$
\frac{d^{2} \Psi}{d z^{2}}=\left\{\begin{array}{c}
\frac{\varepsilon \kappa^{2}}{2 \varepsilon_{\text {int }}} \frac{\mathrm{e}^{\Psi-\alpha_{-}}-\mathrm{e}^{-\Psi-\alpha_{+}}}{1+(\nu / 2)\left(\mathrm{e}^{-\Psi-\alpha_{+}}+\mathrm{e}^{\Psi-\alpha_{-}}-2\right)} \\
\text { for } 0<z<z_{\varepsilon}, \\
\frac{\kappa^{2} \sinh \Psi}{1+\nu(\cosh \Psi-1)} \text { for } z>z_{\varepsilon},
\end{array}\right.
$$


where $\Psi=e \psi / k_{\mathrm{B}} T$ and $\kappa^{-1}=\sqrt{\varepsilon \varepsilon_{0} k_{\mathrm{B}} T / 2 e^{2} c_{0}}$ is the Debye length. Without steric effects $(\nu=0)$, eq 8 simplifies to

$$
\frac{d^{2} \Psi}{d z^{2}}= \begin{cases}\frac{\varepsilon \kappa^{2}}{2 \varepsilon_{\text {int }}}\left(\mathrm{e}^{\Psi-\alpha_{-}}-\mathrm{e}^{-\Psi-\alpha_{+}}\right) & \text {for } 0<z<z_{\varepsilon} \\ \kappa^{2} \sinh \Psi & \text { for } z>z_{\varepsilon} .\end{cases}
$$

\section{Parameterization of the box models}

The introduction of the simplified interfacial box models (eqs 6 and 7) enables the analytical solution of the extended PB equation. The parameters of the box profiles can be obtained by fitting the model results to experimental data, as will be explained in detail below. As a check of the fitting results, we also estimate the parameters based on the interfacial dielectric profile and the ionic potentials of mean force which have been obtained previously by MD simulations. ${ }^{24,28,29}$ We use this second independent parameterization to guarantee the consistency of our approach, and to facilitate the interpretation of the fitted box profiles in terms of the molecular interfacial structure. The full dielectric profile $\varepsilon_{\perp}(z)$ is an oscillating function for diamond surfaces. ${ }^{28,29}$ The potential of mean force exhibits either an attractive minimum or a monotonic repulsive decrease, depending on ion and surface type. ${ }^{27,62}$ From the full dielectric profile, we determine $z_{\varepsilon}$ and $\varepsilon_{\text {int }}$ as follows. ${ }^{28,29}$ Using that the displacement field is constant along the $z$ direction (perpendicular to the interface) in the absence of free charges, the differential capacitance $C$ between $z=0$ and $z=z_{\mathrm{b}}$ (in the aqueous bulk phase far from the surface) is given in terms of $\varepsilon_{\perp}(z)$ by

$$
\frac{1}{C}=\int_{0}^{z_{\mathrm{b}}} \frac{d z}{\varepsilon_{\perp}(z) \varepsilon_{0}}
$$

If we use the box profile (eq 6) for $\varepsilon_{\perp}(z)$, we obtain

$$
\frac{1}{C}=\frac{z_{\varepsilon}}{\varepsilon_{\text {int }} \varepsilon_{0}}+\frac{z_{\mathrm{b}}-z_{\varepsilon}}{\varepsilon \varepsilon_{0}}
$$


Equating eqs 10 and 11 leads to

$$
z_{\varepsilon}=\int_{0}^{z_{\mathrm{b}}} \frac{\varepsilon_{\perp}(z)^{-1}-\varepsilon^{-1}}{\varepsilon_{\mathrm{int}}^{-1}-\varepsilon^{-1}} d z
$$

Similarly, the potential of mean force determines the excess number of ions per unit surface area $\Gamma^{ \pm}$, defined as

$$
\Gamma^{ \pm}=\int_{0}^{z_{\mathrm{b}}}\left(c_{0} \mathrm{e}^{\mp \Psi(z)-\mu_{0}^{ \pm}(z)}-c_{0}\right) d z
$$

For a neutral surface in contact with an infinitely dilute electrolyte, the electrostatic contribution $\Psi(z)$ in eq 13 vanishes, and with the box profile of eq 7 for $\mu_{0}^{ \pm}(z)$ we obtain

$$
\Gamma^{ \pm}=c_{0}\left(\mathrm{e}^{-\alpha_{ \pm}}-1\right) z_{\alpha}^{ \pm}
$$

Equating eqs 13 and 14 yields

$$
z_{\alpha}^{ \pm}=\int_{0}^{z_{\mathrm{b}}} \frac{\mathrm{e}^{-\mu_{0}^{ \pm}(z)}-1}{\mathrm{e}^{-\alpha_{ \pm}}-1} d z .
$$

Obviously, eqs 12 and 15 are insufficient to determine all free parameters $z_{\varepsilon}, \varepsilon_{\text {int }}, z_{\alpha}^{ \pm}$and $\alpha_{ \pm}$. Moreover, we do not have the full dielectric profiles nor the potentials of mean force for all pairs of surfaces and electrolytes. In this paper, therefore, we consider only the case of $z_{\alpha}^{ \pm}=z_{\varepsilon}$ to reduce the number of parameters, and we set $z_{\varepsilon}=0.1 \mathrm{~nm}$, which has been extracted from MD simulations as the width of the low-dielectric layer for hydrophilic surfaces. ${ }^{29,29}$ Furthermore, we consider these interfacial parameters to remain constant when we vary the titrated surface charge density $\sigma_{0}$ and the bulk salt concentration $c_{0}$. This means that we neglect the dielectric decrement due to a finite salt concentration ${ }^{63,64}$ which has been shown to affect the nonlinear capacitance. ${ }^{46,47}$ 


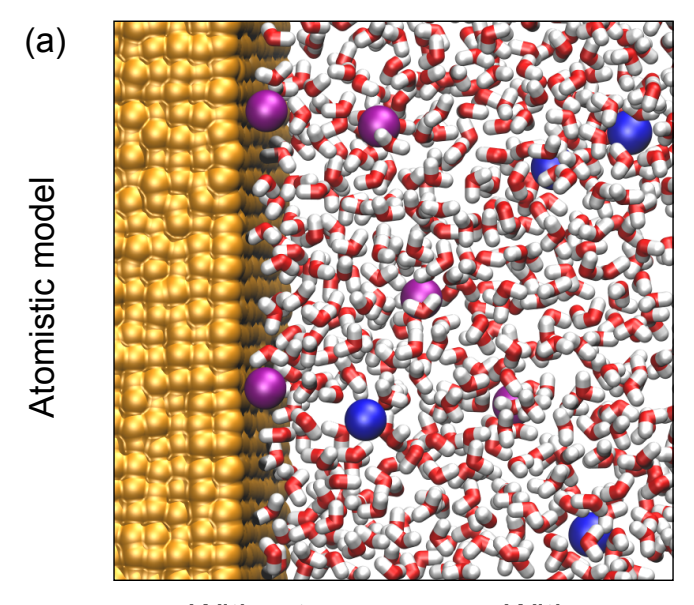

Without steric effect

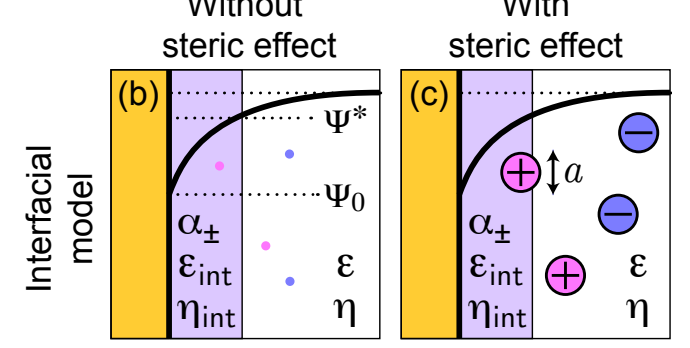

With

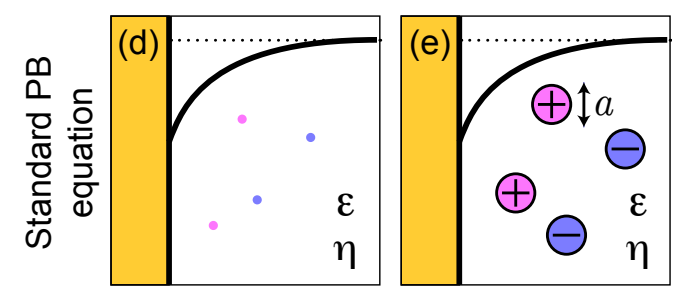

Figure 2: Illustration of the different models studied, including sketches of the electrostatic potential $\Psi(z)$ in panels (b-e). The surface always carries a surface charge density $\sigma_{0}$. A representative snapshot of a molecular simulation of water and ions at a solid surface is shown in (a). The interfacial layer $\left(0<z<z_{\varepsilon}=z_{\alpha}^{ \pm}\right)$is indicated by the shaded purple area in panels (b-c). The ion diameter is denoted by $a$, the parameters $\varepsilon_{\text {int }}, \alpha_{ \pm}$and $\eta_{\text {int }}$ denote the dielectric constant, the nonelectrostatic contribution to the potential and the viscosity in the interfacial layer, respectively. The standard Poisson-Boltzmann (PB) model with bulk dielectric constant $\varepsilon$ and bulk viscosity $\eta$ is shown in panels (d-e). 


\section{Exact solution and the effects of steric interactions}

In this section, we present an exact solution to the extended PB equation introduced in the previous section, and study the influence of steric interactions between the ions on the electrostatic potential. First, we give an overview of the different models we discuss in the remainder of this paper, illustrations of which are shown in figure 2. A snapshot of the molecular structure of a solid-electrolyte interface is shown in figure 2a. The model introduced in the previous section with $\varepsilon_{\text {int }} \neq \varepsilon$ and finite interfacial affinities $\alpha_{ \pm} \neq 0$ we call the interfacial box model (figure $2 \mathrm{bc}$ ), ${ }^{60}$ for which we will eventually also include interfacial viscosity effects. We call the model with $\varepsilon_{\text {int }}=\varepsilon$ and $\alpha_{ \pm}=0$ the standard PB equation (figure 2de). For each of these models, we consider the cases with and without steric effects.

\section{Exact solution without steric effects}

We derive the exact solution of the interfacial box model without steric effects (figure 2c). The solution of eq 9 in the range $z>z_{\varepsilon}$ is ${ }^{2}$

$$
\Psi(z)=2 \ln \frac{1+\mathrm{e}^{-\kappa\left(z-z_{\varepsilon}\right)} \tanh \left(\Psi^{*} / 4\right)}{1-\mathrm{e}^{-\kappa\left(z-z_{\varepsilon}\right)} \tanh \left(\Psi^{*} / 4\right)} \text { for } z>z_{\varepsilon}
$$

where $\Psi^{*}=\Psi\left(z_{\varepsilon}\right)$ is the interfacial potential (see figure 2c). What remains to be solved is eq 9 in the interfacial layer $\left(0<z<z_{\varepsilon}\right)$. Multiplying by $d \Psi / d z$ and integrating once, we obtain

$$
\frac{d}{d z}\left[\frac{1}{2}\left(\frac{d \Psi}{d z}\right)^{2}-\frac{\kappa^{2} \varepsilon}{2 \varepsilon_{\text {int }}}\left(\mathrm{e}^{\Psi-\alpha_{-}}+\mathrm{e}^{-\Psi-\alpha_{+}}\right)\right]=0,
$$

which means that the part in square brackets equals an integration constant. Using this fact, we define $p$ by

$$
\frac{2 \kappa^{2} \varepsilon \chi_{+}}{\varepsilon_{\mathrm{int}}}\left[p-\frac{1}{2}\right]=\frac{1}{2}\left[\frac{d \Psi}{d z}\right]^{2}-\frac{\kappa^{2} \varepsilon}{2 \varepsilon_{\mathrm{int}}}\left(\mathrm{e}^{\Psi-\alpha_{-}}+\mathrm{e}^{-\Psi-\alpha_{+}}\right)
$$


where the symmetric affinity parameter is defined as $\chi_{+}=\exp \left[-\left(\alpha_{+}+\alpha_{-}\right) / 2\right]$. Using the exact solution for $z>z_{\varepsilon}$ (eq 16), we know that $\Psi^{*}$ satisfies $(d \Psi / d z)_{z=z_{\varepsilon}+0}^{2}=2 \kappa^{2}\left(\cosh \Psi^{*}-1\right)$. Inserting this expression into the boundary condition $\varepsilon_{\text {int }} d \Psi /\left.d z\right|_{z=z_{\varepsilon}-0}=\varepsilon d \Psi /\left.d z\right|_{z=z_{\varepsilon}+0}$, we arrive at

$$
\left(\frac{d \Psi}{d z}\right)_{z_{\varepsilon}-0}^{2}=2\left(\frac{\varepsilon}{\varepsilon_{\mathrm{int}}}\right)^{2} \kappa^{2}\left(\cosh \Psi^{*}-1\right) .
$$

Thus, the constant $p$ can be written as a function of $\Psi^{*}$,

$$
p=\frac{\varepsilon}{2 \chi_{+} \varepsilon_{\text {int }}}\left(\cosh \Psi^{*}-1\right)-\frac{1}{4}\left(\chi_{-}^{-1} \mathrm{e}^{\Psi^{*}}+\chi_{-} \mathrm{e}^{-\Psi^{*}}-2\right) .
$$

where the antisymmetric affinity parameter is defined as $\chi_{-}=\exp \left[-\left(\alpha_{+}-\alpha_{-}\right) / 2\right]$.

Our aim is now to obtain a general solution of eq 18 and to determine $\Psi^{*}$ based on the boundary condition at $z=0$, given by $d \Psi /\left.d z\right|_{z=0}=-e \sigma_{0} / \varepsilon_{\text {int }} \varepsilon_{0} k_{\mathrm{B}} T$ (charge neutrality). Using Jacobian elliptic functions, ${ }^{65}$ the general solution of eq 18 for $0<z<z_{\varepsilon}$ equals

$$
\Psi(z)=\left\{\begin{array}{l}
\ln \frac{1-\phi_{\chi_{-}} \operatorname{dn}\left(x_{p} \mid m_{p}\right)}{1+\phi_{\chi_{-}} \operatorname{dn}\left(x_{p} \mid m_{p}\right)}-\frac{\alpha_{+}-\alpha_{-}}{2}, \text { for } p<0, \\
\ln \frac{1-\operatorname{cn}\left(x_{p} \mid m_{p}\right)}{1+\operatorname{cn}\left(x_{p} \mid m_{p}\right)}-\frac{\alpha_{+}-\alpha_{-}}{2}, \text { for } 0 \leq p \leq 1, \\
\ln \frac{1-\operatorname{sn}\left(x_{p} \mid m_{p}\right)}{1+\operatorname{sn}\left(x_{p} \mid m_{p}\right)}-\frac{\alpha_{+}-\alpha_{-}}{2}, \text { for } 1<p,
\end{array}\right.
$$

where $\phi_{\chi_{-}}=\operatorname{sgn}\left(\ln \chi_{-}\right)$, and the index $0 \leq m_{p} \leq 1$ of the Jacobian elliptic functions is defined by

$$
m_{p}=\left\{\begin{array}{lll}
(1-p)^{-1}, & \text { for } \quad p<0 \\
1-p, & \text { for } \quad 0 \leq p \leq 1 \\
1-p^{-1}, & \text { for } \quad 1 \leq p
\end{array}\right.
$$

A detailed derivation is given in the Supporting Information. The index $m_{p}$ of the Jacobian elliptic functions will be omitted in the remainder of this paper. The arguments $x_{p}(z)$ of the 
Jacobian elliptic functions in eq 21 are defined by

$$
\begin{aligned}
& x_{p}(z)= \\
& \left\{\begin{array}{l}
-\phi_{\chi_{-}} \phi_{\Psi^{*}} \kappa_{p}\left(z-z_{\varepsilon}\right)+\mathrm{dn}^{-1}\left(\phi_{\chi_{-}} \omega^{*}\right), \text { for } p<0, \\
-\phi_{\Psi^{*}} \kappa_{p}\left(z-z_{\varepsilon}\right)+\mathrm{cn}^{-1}\left(\omega^{*}\right), \text { for } 0 \leq p \leq 1, \\
\phi_{\Psi^{*}} \kappa_{p}\left(z-z_{\varepsilon}\right)+\operatorname{sn}^{-1}\left(\omega^{*}\right), \text { for } 1<p,
\end{array}\right.
\end{aligned}
$$

where $\omega^{*}=\left(\chi_{-}-\mathrm{e}^{\Psi^{*}}\right) /\left(\chi_{-}+\mathrm{e}^{\Psi^{*}}\right), \phi_{\Psi^{*}}=\operatorname{sgn}\left(\Psi^{*}\right)$, and $\kappa_{p}$ is given by

$$
\kappa_{p}= \begin{cases}\sqrt{1-p} \kappa \sqrt{\chi_{+} \varepsilon / \varepsilon_{\mathrm{int}}}, & \text { for } p<0 \\ \kappa \sqrt{\chi_{+} \varepsilon / \varepsilon_{\mathrm{int}}}, & \text { for } 0 \leq p \leq 1 \\ \sqrt{p} \kappa \sqrt{\chi_{+} \varepsilon / \varepsilon_{\mathrm{int}}}, & \text { for } 1<p\end{cases}
$$

The values of $\Psi^{*}$ and $p$ are determined by the electroneutrality boundary condition as

$$
-\frac{e \sigma_{0}}{\varepsilon_{\mathrm{int}} \varepsilon_{0} k_{\mathrm{B}} T}= \begin{cases}-2 \phi_{\Psi^{*}} \kappa_{p} \mathrm{cs}\left(x_{p}(0)\right), & \text { for } p<0 \\ -2 \phi_{\Psi^{*}} \kappa_{p} \mathrm{ds}\left(x_{p}(0)\right), & \text { for } 0 \leq p \leq 1 \\ -2 \phi_{\Psi^{*}} \kappa_{p} \mathrm{dc}\left(x_{p}(0)\right), & \text { for } 1<p\end{cases}
$$

The electrostatic potential of eq 21 forms the basis of our treatment of the interfacial box model without steric effects in the following sections. General solutions of the PB equation

in terms of Jacobian elliptic functions have been reported previously in the literature, ${ }^{66,67}$ but the combined effects of an interfacial low-dielectric layer and interfacial ion adsorption have not been treated analytically before except in ref 61 .

\section{Linear analysis including steric effects}

In order to include steric effects in the model while keeping the model analytically tractable, we linearize the interfacial box model (figure 2c). Note that linearization of the standard $\mathrm{PB}$ equation (figure 2e) eliminates the effect of steric interactions, but in the presence of ion 
adsorption the steric effect survives even in the linear limit. If we assume $|\Psi(z)| \ll 1$ for all $z$, we can linearize $\mathrm{e}^{ \pm \Psi}$. Such a situation is realized in the case of $\left|\alpha_{+}-\alpha_{-}\right| \ll 1$. After linearization, eq 9 in the presence of steric effects becomes

$$
\frac{d^{2} \Psi}{d z^{2}}=\left\{\begin{array}{l}
\kappa_{\text {int }}^{2}\left(\Psi-\Psi_{\text {int }}\right), \text { for } 0<z<z_{\varepsilon} \\
\kappa^{2} \Psi, \text { for } z>z_{\varepsilon},
\end{array}\right.
$$

where $\kappa_{\text {int }}(>0)$ is defined as

$$
\kappa_{\text {int }}^{2}=\frac{\varepsilon \kappa^{2}}{2 \varepsilon_{\text {int }}} \frac{\left(\mathrm{e}^{-\alpha_{+}}+\mathrm{e}^{-\alpha_{-}}\right)(1-\nu)+2 \nu \mathrm{e}^{-\alpha_{+}-\alpha_{-}}}{\left[1+(\nu / 2)\left(\mathrm{e}^{-\alpha_{+}}+\mathrm{e}^{-\alpha_{-}}-2\right)\right]^{2}},
$$

and

$$
\Psi_{\text {int }}=\frac{\left(\mathrm{e}^{-\alpha_{+}}-\mathrm{e}^{-\alpha_{-}}\right)\left[1+(\nu / 2)\left(\mathrm{e}^{-\alpha_{+}}+\mathrm{e}^{-\alpha_{-}}-2\right)\right]}{\left(\mathrm{e}^{-\alpha_{+}}+\mathrm{e}^{-\alpha_{-}}\right)(1-\nu)+2 \nu \mathrm{e}^{-\alpha_{+}-\alpha_{-}}} .
$$

The solution of eq 26 can be written as a linear combination of exponential functions,

$$
\Psi(z)= \begin{cases}A \mathrm{e}^{-z \kappa_{\mathrm{int}}}+B \mathrm{e}^{z \kappa_{\mathrm{int}}}+\Psi_{\mathrm{int}}, & \text { for } 0<z<z_{\varepsilon} \\ D \mathrm{e}^{-z \kappa}, & \text { for } z>z_{\varepsilon}\end{cases}
$$

With the boundary conditions $\varepsilon_{\text {int }} d \Psi /\left.d z\right|_{z=z_{\varepsilon}-0}=\varepsilon d \Psi /\left.d z\right|_{z=z_{\varepsilon}+0},\left.\Psi\right|_{z=z_{\varepsilon}-0}=\left.\Psi\right|_{z=z_{\varepsilon}+0}$, and $d \Psi /\left.d z\right|_{z=0}=-e \sigma_{0} / \varepsilon_{\text {int }} \varepsilon_{0} k_{\mathrm{B}} T$, we determine the coefficients $A, B$, and $D$ to be

$$
\begin{aligned}
A & =\frac{1}{1+G}\left(\frac{e \sigma_{0}}{\varepsilon_{\mathrm{int}} \varepsilon_{0} k_{\mathrm{B}} T \kappa_{\mathrm{int}}}-\frac{\varepsilon \kappa \mathrm{e}^{-z_{\varepsilon} \kappa_{\mathrm{int}}} \Psi_{\mathrm{int}}}{\varepsilon \kappa+\varepsilon_{\mathrm{int}} \kappa_{\mathrm{int}}}\right), \\
B & =\frac{G}{1+G}\left(-\frac{e \sigma_{0}}{\varepsilon_{\mathrm{int}} \varepsilon_{0} k_{\mathrm{B}} T \kappa_{\mathrm{int}}}-\frac{\varepsilon \kappa \mathrm{e}^{z_{\varepsilon} \kappa_{\mathrm{int}}} \Psi_{\mathrm{int}}}{\varepsilon \kappa-\varepsilon_{\mathrm{int}} \kappa_{\mathrm{int}}}\right), \\
D & =A \mathrm{e}^{z_{\varepsilon}\left(\kappa-\kappa_{\mathrm{int}}\right)}+B \mathrm{e}^{z_{\varepsilon}\left(\kappa+\kappa_{\mathrm{int}}\right)}+\mathrm{e}^{z_{\varepsilon} \kappa} \Psi_{\mathrm{int}},
\end{aligned}
$$

where

$$
G=\frac{\varepsilon \kappa-\varepsilon_{\text {int }} \kappa_{\text {int }}}{\varepsilon \kappa+\varepsilon_{\text {int }} \kappa_{\text {int }}} \mathrm{e}^{-2 z_{\varepsilon} \kappa_{\text {int }}}
$$


Eqs 29-33 constitute an analytical solution to the extended PB equation in the presence of steric interactions, valid for small electrostatic potentials. However, for medium-sized ions, $a=0.3 \mathrm{~nm}$, the steric effects become important only when $\nu \mathrm{e}^{-\alpha_{ \pm}} \approx 1$, which happens for large adsorption potentials, for example, $\alpha_{ \pm} \approx-3$ for $c_{0}=1 \mathrm{M}$. Because the case of large adsorption potentials, while keeping $\left|\alpha_{+}-\alpha_{-}\right| \ll 1$, is unrealistic for common electrolytes, we will study the effects of steric interactions numerically for the nonlinear PB equation only, at the end of this section. Now, we will focus instead on the low salinity limit $\kappa z_{\varepsilon} \ll 1$, where steric effects can be neglected $(\nu=0)$. Expanding the surface potential $\Psi_{0}=\Psi(0)$ with respect to $\kappa z_{\varepsilon}$, we obtain to first order in $\kappa z_{\varepsilon}$

$$
\begin{aligned}
\Psi_{0}= & \frac{e \sigma_{0}}{\varepsilon \varepsilon_{0} k_{\mathrm{B}} T \kappa}\left[1+z_{\varepsilon} \kappa\left(\frac{\varepsilon}{\varepsilon_{\text {int }}}-\frac{\mathrm{e}^{-\alpha_{+}}+\mathrm{e}^{-\alpha_{-}}}{2}\right)\right] \\
& +z_{\varepsilon} \kappa \frac{\mathrm{e}^{-\alpha_{+}}-\mathrm{e}^{-\alpha_{-}}}{2}+\mathcal{O}\left(\kappa^{2} z_{\varepsilon}^{2}\right),
\end{aligned}
$$

and the interfacial boundary potential becomes

$$
\begin{aligned}
\Psi^{*}= & \frac{e \sigma_{0}}{\varepsilon \varepsilon_{0} k_{\mathrm{B}} T \kappa}\left[1-z_{\varepsilon} \kappa \frac{\mathrm{e}^{-\alpha_{+}}+\mathrm{e}^{-\alpha_{-}}}{2}\right] \\
& +z_{\varepsilon} \kappa \frac{\mathrm{e}^{-\alpha_{+}}-\mathrm{e}^{-\alpha_{-}}}{2}+\mathcal{O}\left(\kappa^{2} z_{\varepsilon}^{2}\right) .
\end{aligned}
$$

The last terms in eqs 34 and 35 contain the increase of the potential due to asymmetric specific ion adsorption.

\section{Asymptotic analysis for high surface charge}

We now consider the asymptotic behavior of the nonlinear PB equation in the limit of $\sigma_{0} \rightarrow \pm \infty$ for the interfacial box model without steric effects (figure $2 \mathrm{~b}$ ). In this limit, $\Psi^{*}$ (determined from eqs 23, 24 and 25) approaches a constant value for any specific adsorption strength, independent of the salt concentration. This means that a given charge increment on the surface is totally compensated for by the increase of the charge density within the 
interfacial layer $\left(z<z_{\varepsilon}\right)$, because any other distribution of the extra counterions would change $\Psi^{*}$. We restrict our discussion to the cases in which $p$, defined in eq 18 , is much higher than unity. The detailed derivation of the asymptotic equations is given in the Supporting Information. Further analysis of eqs 21 and 25 yields the boundary potential in the limit of high surface charge and low salinity

$$
\Psi^{*} \approx \pm \ln \left[\frac{4 \operatorname{arccosh}^{2} \sqrt{\mathrm{e}^{\alpha_{\mp} \varepsilon / \varepsilon_{\mathrm{int}}}}}{\left(\varepsilon / \varepsilon_{\mathrm{int}}\right) z_{\varepsilon}^{2} \kappa^{2}\left(\varepsilon / \varepsilon_{\mathrm{int}}-\mathrm{e}^{-\alpha_{\mp}}\right)}\right]
$$

and the surface potential in the limit of high surface charge density

$$
\Psi_{0} \approx \pm \ln \left[\frac{\mathrm{e}^{\alpha_{\mp}} \varepsilon_{\text {int }}}{\kappa^{2} \varepsilon}\left(\frac{e \sigma_{0}}{\varepsilon_{\mathrm{int}} \varepsilon_{0} k_{\mathrm{B}} T}\right)^{2}\right]
$$

where the \pm in front of the logarithm correspond to the limits of $\sigma_{0} \rightarrow \infty$ and $\sigma_{0} \rightarrow-\infty$, respectively. Note that eqs 36 and 37 depend on the ion-specific interaction parameter $\alpha_{ \pm}$ of the counterion only. For example, in the limit of $\sigma_{0} \rightarrow \infty, \Psi^{*}$ and $\Psi_{0}$ depend only on $\alpha_{-}$, not on $\alpha_{+}$. This is a direct consequence of the exclusion of the coions from the interfacial layer. Finally, we remark that eqs 36 and 37 are also valid for the homogeneous dielectric case $\left(\varepsilon_{\text {int }}=\varepsilon\right)$. Using the relation $\operatorname{arccosh} \sqrt{x} \approx \sqrt{x-1}$ in the limit $x \rightarrow 1$, eq 36 with $\varepsilon_{\text {int }}=\varepsilon$ and $\alpha_{ \pm}=0$ becomes

$$
\Psi^{*} \approx \pm 2 \ln \frac{2}{\kappa z_{\varepsilon}},
$$

and eq 37 becomes

$$
\Psi_{0} \approx \pm 2 \ln \frac{e \sigma_{0}}{\varepsilon \varepsilon_{0} k_{\mathrm{B}} T \kappa} .
$$

Eqs 38 and 39 correctly recover the expressions which follow from the exact solution of the standard PB equation. ${ }^{2}$

Figure 3 shows $\Psi^{*}$ for different salt concentrations as a function of the surface charge density $\sigma_{0}$ without steric effects. In figure $3 \mathrm{a}$, the solid lines denote the full analytic solution given by eq 25, and the dotted lines denote the linearized solution given by eq $29^{61}$ for 

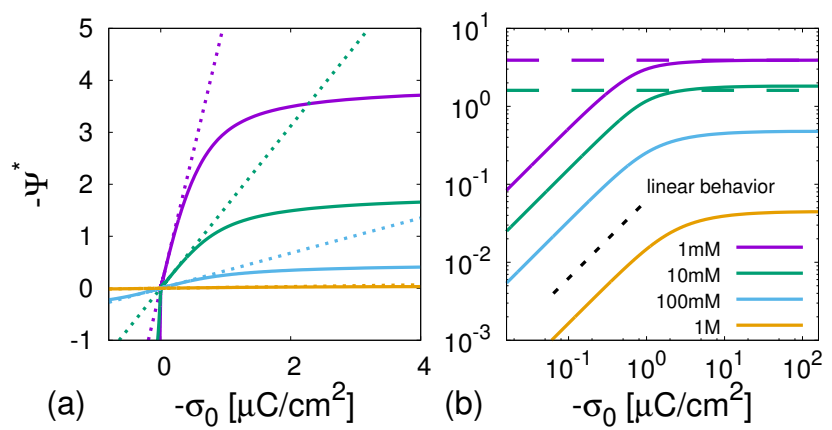

Figure 3: Interfacial boundary potential $\Psi^{*}$ as a function of the titrated surface charge $\sigma_{0}$, eq 25, (solid lines) on (a) linear and (b) double-logarithmic scales. We use the parameters $\varepsilon=78,{ }^{68} T=298 \mathrm{~K}, \varepsilon_{\text {int }}=1, z_{\varepsilon}=0.1 \mathrm{~nm}$, and $\alpha_{ \pm}=0$ without steric effects. Dotted lines in (a) are calculated by linear analysis eq $29,{ }^{61}$ and broken lines in (b) denote the asymptotic result eq 36 for $1 \mathrm{mM}$ and $10 \mathrm{mM}$.
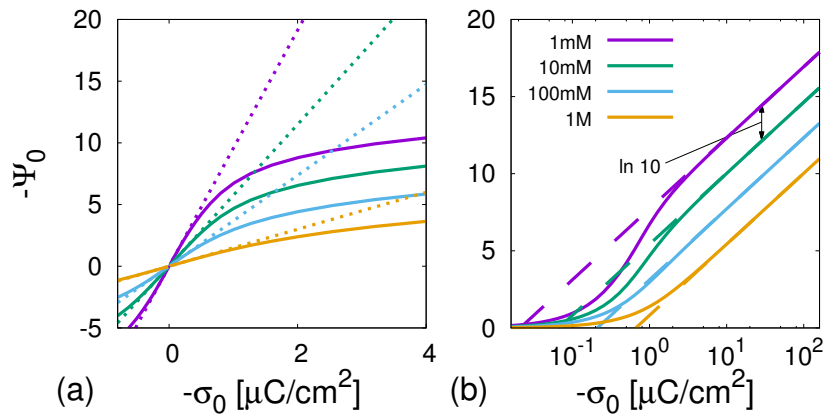

Figure 4: The surface potential $\Psi_{0}$ as a function of the titrated surface charge density $\sigma_{0}$ (solid lines) on (a) linear and (b) semi-logarithmic scales. We use the parameters $\varepsilon=78,{ }^{68}$ $T=298 \mathrm{~K}, \varepsilon_{\text {int }}=1, z_{\varepsilon}=0.1 \mathrm{~nm}$, and $\alpha_{ \pm}=0$ without steric effects. Dotted lines in (a) are calculated by linear analysis eq 29, ${ }^{61}$ and broken lines in (b) denote eq 37 . The broken lines in (b) increase by $\ln 10$ when the salinity increases by a factor 10 , indicating that $\psi_{0}$ depends logarithmically on $c_{0}$. 
parameters $\varepsilon=78,{ }^{68} T=298 \mathrm{~K}, \varepsilon_{\mathrm{int}}=1, z_{\varepsilon}=0.1 \mathrm{~nm}$, and $\alpha_{ \pm}=0$. For weakly charged surfaces, $\Psi^{*}$ increases linearly with the surface charge density. However, above a specific $\sigma_{0}$ depending on the salinity, $\Psi^{*}$ shows a nonlinear dependence. Plotting the same curves on a double-logarithmic scale, figure $3 \mathrm{~b}$, clearly shows the saturation of $\Psi^{*}$ in the limit of strongly charged surfaces. Horizontal broken lines indicate eq 36 . The saturation of $\Psi^{*}$ is not specific for the interfacial box model without steric effects, figure $2 \mathrm{~b}$, but also occurs for the standard PB equation without steric effects, figure $2 \mathrm{~d} .{ }^{2}$ Figure 4 shows the surface potential $\Psi_{0}$ at a given salt concentration without steric effects. In figure 4a (linear scale), the solid lines denote the numerical result obtained by solving eqs 21 and 25, and the dotted lines denote the linearized solution eq 29. ${ }^{61}$ The broken lines in figure $4 \mathrm{~b}$, which show $\Psi_{0}$ on a semi-logarithmic scale, indicate the logarithmic dependence $\left|\Psi_{0}\right| \sim \ln \left(\sigma_{0}^{2} / c_{0}\right)$ for large $\left|\sigma_{0}\right|$, which follows from eq 37 .

Finally, we examine the effects of steric interactions on the asymptotic behavior of $\Psi^{*}$ and $\Psi_{0}$ by numerically solving eq 8 . We use the effective ion diameter $a=0.3 \mathrm{~nm}$, corresponding to the ionic diameter of potassium. ${ }^{69}$ The solid lines in figure 5 show $\Psi^{*}$ and $\Psi_{0}$, including steric effects, as a function of $\sigma_{0}$ for different ion concentrations. Comparing figure 5ab with the corresponding curves without steric effects, figure $3 \mathrm{~b}$ and $4 \mathrm{~b}$, respectively, it becomes clear that steric effects induce strong deviations of both $\Psi^{*}$ and $\Psi_{0}$ from the asymptotic limit only at high surface charge density $\left(\left|\sigma_{0}\right| \gg 10 \mu \mathrm{C} / \mathrm{cm}^{2}\right)$. For clarity, the asymptotic equations without steric effects, eqs 36 and 37, are reproduced in figure 5 as broken lines. This comparison shows that for ions with the effective size of potassium, there still is a large range of medium surface charge densities in which the asymptotic behavior predicted by the analytically solvable model is valid, and steric effects are negligible. 

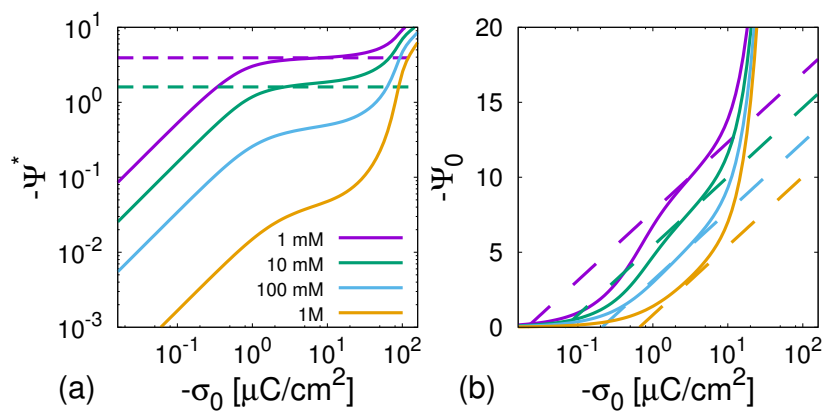

Figure 5: $\Psi^{*}$ and $\Psi_{0}$ as a function of the titrated surface charge density $\sigma_{0}$ (solid lines) including steric effects. We use parameters $\varepsilon=78,{ }^{68} T=298 \mathrm{~K}, \varepsilon_{\mathrm{int}}=1, z_{\varepsilon}=0.1 \mathrm{~nm}$, and $\alpha_{ \pm}=0$. The effective ion diameter is $a=0.3 \mathrm{~nm}$. Broken lines denote eq 36 (a) and eq 37 (b). Panel (a) corresponds to figure $3 \mathrm{~b}$ and panel (b) corresponds to figure $4 \mathrm{~b}$ when steric effects are included.

\section{Differential capacitance}

From the surface potential $\Psi_{0}$ derived in the previous section, we calculate the differential capacitance, and compare it with experimental measurements on carbon- and metal-electrolyte interfaces. The potential variation across a solid-liquid interface, which is caused in the solid by the electron distribution and in the liquid by the ionic distribution and the water polarization, is shown schematically in figure 6. In general, the definition of the total capacitance is

$$
\frac{1}{C}=\frac{d \psi_{0}}{d \sigma_{0}}+\frac{d \psi_{\mathrm{sub}}}{d \sigma_{0}} \equiv \frac{1}{C_{\mathrm{liq}}}+\frac{1}{C_{\mathrm{sub}}}
$$

where $\psi_{\text {sub }}$ is the potential difference between the bulk solid phase and the interface (at $z=0), \psi_{0}$ and $\sigma_{0}$ (defined in the previous section) are the surface potential and the surface charge density, respectively, and $C_{\text {liq }}$ and $C_{\text {sub }}$ denote the capacitances of the liquid and the solid substrate, respectively. Note that we use the potential $\psi(z)=k_{\mathrm{B}} T \Psi(z) / e$ in units of Volt in this section.

To compare with the extended PB equation developed in the previous sections, we first summarize the results of the standard PB equation. From the standard PB equation, ${ }^{70,71}$ 


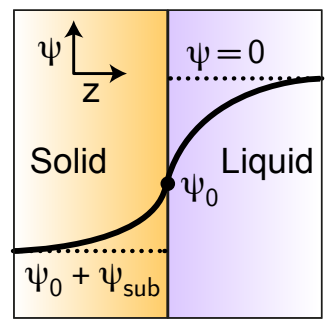

Figure 6: Schematic picture of the typical profile of the electrostatic potential at the solidliquid interface.

the surface charge density in terms of $\psi_{0}$ equals

$$
\sigma_{0}=\frac{2 \varepsilon \varepsilon_{0} k_{\mathrm{B}} T \kappa}{e} \sinh \left(\frac{e \psi_{0}}{2 k_{\mathrm{B}} T}\right)
$$

which is the equivalent of eq 25 in the absence of interfacial effects, i.e. for $\varepsilon_{\text {int }}=\varepsilon$ and $\alpha_{ \pm}=0$. The capacitance of the liquid phase equals

$$
C_{\text {liq }}=\frac{d \sigma_{0}}{d \psi_{0}}=\varepsilon \varepsilon_{0} \kappa \cosh \left(\frac{e \psi_{0}}{2 k_{\mathrm{B}} T}\right) .
$$

We refer to eq 42 as the capacitance of the diffuse layer. At the PZC $\left(\sigma_{0}=0\right.$ and therefore $\left.\psi_{0}=0\right)$, the capacitance of the liquid phase, eq 42 , becomes

$$
C_{\mathrm{PZC}}=\varepsilon \varepsilon_{0} \kappa .
$$

The contribution of the solid phase to the capacitance, $1 / C_{\text {sub }}$, has been considered to be minor for a long time, which is equivalent to assuming perfect conduction in the solid. However, it has been known since the 1980s that the capacitance of the solid phase is typically finite, and its contribution cannot always be neglected. ${ }^{72-78}$ For graphite, the solid phase capacitance is positive $\left(C_{\text {sub }}>0\right)$, decreasing the total capacitance. Our interfacial box model considers only the capacitance of the liquid, and therefore, we add the additional contribution from the solid phase when we compare with the experimental data of carbon, as described below. 


\section{Capacitance at the point of zero charge}

For a surface in contact with an electrolyte, the salt type and concentration, the surface potential and the $\mathrm{pH}$ determine the surface charge density $\sigma_{0}$. The point where $\sigma_{0}=0$ is defined as the point of zero charge (PZC), which is typically expressed as a $\mathrm{pH}$ value. In experiments, the PZC is usually defined by the minimum of the capacitance as a function of the interface potential $\psi_{0}$, based on the standard PB equation (eqs 41 and 42). Unlike eqs 41 and 42, however, the PZC does not correspond to $\psi_{0}=0$ for most combinations of surfaces and electrolytes, partly due to asymmetric ion-surface affinities. Including ion affinities using the interfacial box model, eqs 8 and 9 are symmetric with respect to the transformation $\psi_{0} \rightarrow-\psi_{0}$ only in the case of symmetric ion affinities, $\alpha_{+}=\alpha_{-}$. In this case, therefore, the minimum of the differential capacitance as a function of $\psi_{0}$ exactly corresponds to the PZC indeed. However, in the case of $\alpha_{+} \neq \alpha_{-}$, the minimum is generally not identical to the PZC. ${ }^{79}$ Further discussion is given in the Supporting Information. Here, we consider $\alpha_{+}=\alpha_{-} \equiv \alpha$, in which case we can calculate the capacitance at the PZC from the linear PB equation (eq 29-33) as

$$
\frac{1}{C_{\mathrm{PZC}}}=\frac{1}{\varepsilon_{\mathrm{int}} \varepsilon_{0} \kappa_{\mathrm{int}}}\left(\frac{1-G}{1+G}\right)+\frac{1}{C_{\mathrm{sub}}},
$$

where $G$ is defined in eq 33 . For $C_{\mathrm{PZC}}$, the effects of steric interactions are minor except at high salt concentration $(\nu \lesssim 1)$ and in the case of strong adsorption $(-\alpha \ll-1)$. Therefore, we neglect steric interactions at the PZC, setting $\nu=0$. Assuming a conducting surface $\left(C_{\text {sub }} \rightarrow \infty\right)$, eq 44 is approximated in the low salinity regime as ${ }^{61}$

$$
C_{\mathrm{PZC}} \approx\left[\frac{1}{\varepsilon \varepsilon_{0} \kappa}+\frac{z_{\varepsilon}}{\varepsilon_{\mathrm{int}} \varepsilon_{0}}-\frac{\mathrm{e}^{-\alpha} z_{\varepsilon}}{\varepsilon \varepsilon_{0}}\right]^{-1}, \text { for } \kappa z_{\varepsilon} \ll 1
$$

and in the high salinity regime as

$$
C_{\mathrm{PZC}} \approx \sqrt{\varepsilon \varepsilon_{\mathrm{int}}} \varepsilon_{0} \kappa \mathrm{e}^{-\alpha / 2}, \text { for } \kappa z_{\varepsilon} \gg 1 .
$$


Eq 45 shows that in the low salinity regime, the total capacitance is equivalent to a series circuit of two capacitors: the diffuse layer capacitance $\varepsilon \varepsilon_{0} \kappa$ and the interfacial layer capacitance $C_{\text {int }}=\left(z_{\varepsilon} / \varepsilon_{\text {int }} \varepsilon_{0}-\mathrm{e}^{-\alpha} z_{\varepsilon} / \varepsilon \varepsilon_{0}\right)^{-1}$, which depends on $z_{\varepsilon}$ and $\varepsilon_{\text {int }}$. In the high salinity regime, by contrast, eq 46 cannot be understood as a series of capacitors. Instead, it follows from the standard $\mathrm{PB}$ equation by a transformation of $\varepsilon \rightarrow \varepsilon_{\text {int }}$ and $c_{0} \rightarrow c_{0} \mathrm{e}^{-\alpha}$, so $\kappa \rightarrow \kappa \sqrt{\varepsilon / \varepsilon_{\text {int }}} \mathrm{e}^{-\alpha / 2}$. That means that the ions are predominantly located inside the interfacial layer, where the system behaves as a standard PB system with a dielectric constant $\varepsilon_{\text {int }}$ and a reference concentration $c_{0} \mathrm{e}^{-\alpha}$. For strong attraction $\left(\alpha<-\ln \left(\varepsilon / \varepsilon_{\text {int }}\right)\right)$, the capacitance is larger than the result of the standard $\mathrm{PB}$ equation, eq 43, whereas for weak attraction the interfacial layer makes $C_{\mathrm{PZC}}$ smaller, for any value of $\kappa$.

Figure 7 shows the differential capacitance at the PZC as a function of the salt concentration. The black broken line in figure 7 denotes the capacitance of the standard PB equation, eq 43. We compare the results from the standard PB equation and from our interfacial box model with experimental data for the capacitance of metals (blue symbols), planar carbon (graphite, graphene, and diamond) (red symbols), and porous carbon (black symbols), plotted in figure 7 and summarized in the Supporting Information. The capacitance of metals almost coincides with the result of the standard PB equation, eq 43 (black broken line), and in some cases slightly exceeds the result of the standard PB equation, which has been rationalized by the roughness of the metallic surfaces. ${ }^{33,80}$ In contrast, the planar non-porous carbon data at high salinity fall short of the standard PB prediction. Surprisingly though, the planar carbon data exceed the standard PB equation at low salinity. Due to the indirect measurement method, the capacitance per unit mass of porous carbon does not scale proportional to the surface area per mass,${ }^{38}$ which is discussed in the Supporting Information. 


\section{Variation of the ion-surface interaction $\alpha$}

In our interfacial box model, the capacitance of the interface is decreased by the low-dielectric interfacial layer, depending on its width. The black solid line in figure 7 denotes the case of the interfacial box model with $\alpha=0, \varepsilon_{\text {int }}=1$ and $z_{\varepsilon}=0.1 \mathrm{~nm}$, eq 44 , showing a decrease of the capacitance with respect to the standard $\mathrm{PB}$ equation of about one order of magnitude at high salt concentration. In addition, the ion-surface interaction can increase the capacitance, for attractive potentials $(\alpha<0)$, or decrease the capacitance, for repulsive potentials $(\alpha>0)$. These tendencies can be inferred directly from eqs 45 and 46. The colored lines in figure 7 denote the results of our interfacial box model without steric effects (figure $2 b$ ) for different values of $\alpha$, calculated using eq $44, \varepsilon_{\text {int }}=1$ and $z_{\varepsilon}=0.1 \mathrm{~nm}$. To reproduce the experimental data, we vary the ion-surface interaction $\alpha$ in the interfacial box model according to $\alpha=-4$, $-2,2$, and 4 . The prediction according to strong attraction $(\alpha=-4$, blue solid line) agrees well with the experimental data of metals, and the results of ion-specific repulsion $(\alpha=2$,

orange broken line) agree well with the experimental data of porous carbons, ${ }^{28,29}$ suggesting that the nonelectrostatic interaction is attractive for metals and repulsive for porous carbons. However, even using the strong repulsive interaction $\alpha=4$, the model does not reproduce the capacitance of planar carbons (red data points), such as graphenes and graphites. This fact indicates the necessity of assuming a finite $C_{\text {sub }}$ for planar non-porous carbons, which we will discuss later. The difference between porous and non-porous carbons can be understood by realizing that the electronic structure of porous carbons is more metallic than that of planar non-porous carbons, ${ }^{81,82}$ and thus the capacitance of the liquid phase might dominate $C_{\text {sub }}$ as discussed in the Supporting Information.

\section{Displacement of the image plane - silver surfaces}

An alternative explanation for the experimental data for metals is based on the spill-over of electrons at metallic surfaces. As shown in figure 7 , the predicted capacitance $C_{\text {PzC }}$ with $z_{\varepsilon}=0.1 \mathrm{~nm}, \varepsilon_{\mathrm{int}}=1$, and $\alpha=0$ is an order of magnitude smaller than the one measured 


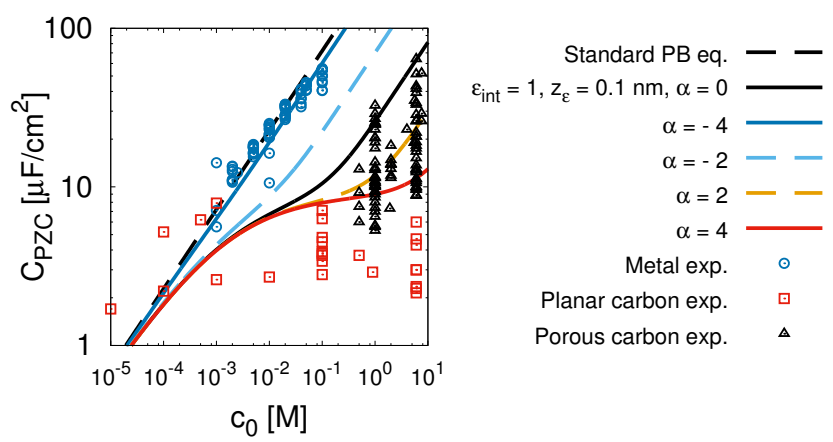

Figure 7: Differential capacitance at the PZC $C_{\text {PZC }}$ as a function of the salt concentration $c_{0}$. The black solid line denotes the interfacial box model with $\alpha=0, \varepsilon_{\text {int }}=1, z_{\varepsilon}=0.1 \mathrm{~nm}$, and $\nu=0$ and the black broken line denotes the standard PB equation. Symbols denote the experimental data for metals (blue), planar carbons (red), and porous carbons (black). Colored solid and broken lines denote the interfacial box model for varying surface affinity $\alpha$.

at metal surfaces. ${ }^{28,29,83,84}$ Additional contributions to the capacitance originating from the excess charge on metallic surfaces have been considered in the literature. ${ }^{73-75,78,85}$ Here, we include the spill-over of electrons, which causes a displacement of the image plane toward the liquid, by adjusting the width of the interfacial layer $z_{\varepsilon}$ to fit the experimental data. As the reference experimental data set, we use the capacitance of a silver (110) surface with a $\mathrm{KPF}_{6}$ (potassium hexafluoro-phosphate) solution. ${ }^{33}$ The blue solid line in figure 8a denotes the interfacial box model result when we adjust $z_{\varepsilon}$ to fit the experimental data (black points) with $\varepsilon_{\text {int }}=1$ and $\alpha=0$ fixed. The resultant interfacial layer width is $z_{\varepsilon}=0.007 \mathrm{~nm}$, which is much thinner than $z_{\varepsilon}=0.1 \mathrm{~nm}$ extracted for diamond surfaces from classical MD simulations with constant surface charge $\mathrm{e}^{28,29}$ and used in figure 7. As a possible explanation for the origin of this discrepancy, we note that the difference between the fitted and predicted widths, $0.093 \mathrm{~nm}$, is of the same order as the displacement of the image plane of metals obtained by quantum-mechanical calculations. ${ }^{73-75,78}$ Therefore, the reduced width of the interfacial layer could be caused by the spill-over of electrons from the metal up to the image plane, which is known to be displaced toward the liquid. 


\section{Substrate capacitance - graphite surfaces}

Using the strong repulsive interaction $\alpha=4$ in figure 7 , the interfacial box model still shows a discrepancy with the experimental data for the capacitance of planar carbon (red symbols in figure 7). One explanation for the very small capacitance of planar carbon is provided by the low density of electronic states at the Fermi level, ${ }^{72,76,78,86,87}$ which causes deep penetration of the external electric field into the substrate. As a result, the surface charge forms a diffuse-layer-like structure on the carbon side, and the image plane is located inside the carbon. Here, we include the contribution from the surface charge distribution in the carbon substrate by adding an effective carbon capacitance $C_{\text {sub }}$ in the series circuit (eq 40). Based on the results of density functional theory simulations, ${ }^{78}$ figure S2b in the Supporting Information, we use the following heuristic expression for the additional effective carbon capacitance

$$
C_{\text {sub }}=\frac{\varepsilon_{0}}{z_{\text {im }}} \mathrm{e}^{\left|\sigma_{0}\right| / \Sigma}
$$

where $z_{\mathrm{im}}>0$ is the displacement of the image plane from the surface of the carbon and $\Sigma$ is an adjustable parameter necessary for finite surface charge. We discuss the validity of eq 47 in the Supporting Information. As experimental reference, we consider the capacitance of stress-annealed pyrolytic graphite, which is essentially non-porous, against a NaF solution

plotted in figure 8b. ${ }^{37}$ Because the experimental capacitance is larger than the standard PB result in figure $8 \mathrm{~b}$ for low salinity, eq 47 does not explain the carbon capacitance in the low salinity limit. Therefore, we adjust both $\alpha$ and $z_{\text {im }}$ to fit the experimental data with $\varepsilon_{\text {int }}=1$ and $z_{\varepsilon}=0.1 \mathrm{~nm}$ fixed. The red solid line in figure $8 \mathrm{~b}$ is the result of the interfacial box model with the adsorption energy $\alpha=-8.5$, and $z_{\mathrm{im}}=0.32 \mathrm{~nm}$ (corresponding to $\left.C_{\text {sub }}=2.8 \mu \mathrm{F} / \mathrm{cm}^{2}\right)$. At about half the value, $C_{\text {sub }}$ is in reasonable agreement with a density functional theory estimate. ${ }^{78}$ Note that $\Sigma$ is not needed since $\sigma_{0}=0$. Agreement with the experimental data is excellent for both low and high salinity. However, the resultant ionspecific parameter $\alpha=-8.5$ is strongly attractive, whereas the potentials of mean force at 
different surface types calculated by MD simulations are either repulsive (fluoride) or never exceed $-0.5 k_{\mathrm{B}} T$ (sodium). ${ }^{27} \mathrm{~A}$ strong surface affinity is typically observed, however, for organic surfactants. ${ }^{88,89}$ Therefore, the presence of charged organic impurities might explain the good agreement found in figure 8b.
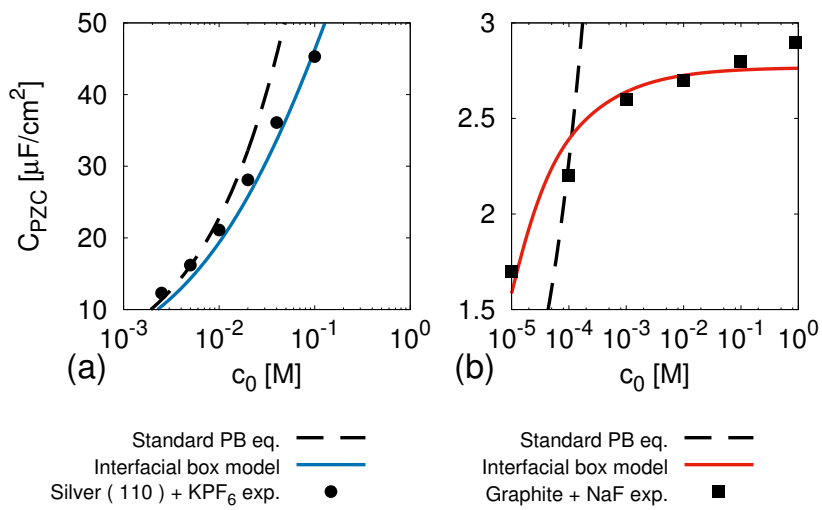

Figure 8: Differential capacitance at the PZC as a function of the salt concentration. The black broken line denotes the standard PB equation eq 43. Black symbols in (a) denote the experimental data for a silver (110) surface in contact with a $\mathrm{KPF}_{6}$ solution. ${ }^{33}$ The solid line in (a) denotes the interfacial box model without steric effects, using the adjusted $z_{\varepsilon}=0.007 \mathrm{~nm}$ in eq 44 . Black symbols in (b) denote the experimental data for stressannealed pyrolytic graphite surface in contact with a NaF solution. ${ }^{37}$ The solid line in (b) denotes the interfacial box model without steric effects, using the adjusted $z_{\mathrm{im}}=0.32 \mathrm{~nm}$ and $\alpha=-8.5$ in eqs (44), (40) and (47).

Summarizing, the capacitance of metal surfaces can be explained by the interfacial box model assuming either an attractive ion-surface potential (figure 7), or a reduced width of the interfacial layer due to the spill-over of electrons, figure 8a. Which of these phenomena dominates the behavior of the metal-electrolyte interface remains to be determined, for example by explicitly calculating the ionic surface affinity at metallic surfaces. The lowdielectric interfacial layer also explains the capacitance of porous carbon surfaces using the parameters extracted from simulations, as noted earlier, ${ }^{28}$ whereas it does not explain the capacitance of planar non-porous carbon, for which we have to assume a finite substrate capacitance. This fact might be caused by the different electronic structures of porous and planar carbon. ${ }^{81,82}$ 


\section{Differential capacitance at finite surface charge}

\section{Comparison with experimental data of silver}

The dependence of the interfacial capacitance on the surface potential is highly nonlinear due to the specific interactions between the ions, the water and the solid substrate. It is well-known that steric effects are essential to understand the differential capacitance at finite charge because of the increased ion concentration near the interface. ${ }^{16,17,19,43-47,49}$ Here, we study the same systems as the ones we considered in the previous section, silver and graphite, away from the PZC. In figure 9a, we show the differential capacitance (the capacitance as a function of the surface potential measured by $\mathrm{AC}$ voltammetry or impedance spectroscopy) of the interface between silver (110) and a $\mathrm{KPF}_{6}$ solution as a function of the surface potential for various salt concentrations. The experimental data shows two maxima which correspond to the voltages at which the counterion concentration reaches its maximum packing density near the surface. ${ }^{44}$ The absolute voltages of the maxima on the positive and negative sides are almost equal, whereas the height of the maximum is slightly larger on the positive side compared to the negative side. The near symmetry is remarkable because $\mathrm{PF}_{6}{ }^{-}$is a polyatomic anion which is very different from the mono-atomic cation $\mathrm{K}^{+}$. We calculate the capacitance of the interfacial box model including steric effects (figure 2c). To fit the location of the maxima in the capacitance curves, we adjust the effective ion diameter. Note that we use a single effective diameter for both ions. In the Supporting Information, we discuss the effect of varying the ion diameter in detail. The other parameters are the same as those used in figure 8a. We find quantitative agreement with the experimental data regarding both the voltage dependence (being parabolic at low voltages, and reproducing the maximum and decay at high voltages) and the salinity dependence. The quantitative agreement is extraordinary: although the qualitative behavior has been discussed intensely, very

few studies have considered a quantitative comparison with experiments. ${ }^{45,49}$ The effective diameter $a=1.2 \mathrm{~nm}$ resulting from the fit is larger than the largest diameter $a=0.59 \mathrm{~nm}$ 
of the octahedral shape of $\mathrm{PF}_{6}{ }^{-} .{ }^{90}$ This large effective diameter shows that the definition of an ionic radius from the ionic crystal structure is not meaningful for the highly concentrated counterion situation near the surface. ${ }^{63,64}$ The decrement of the local dielectric constant due to the local counterion concentration also contributes to the differential capacitance, affecting the resulting estimates of the ion diameter. ${ }^{46,47}$

\section{Comparison with experimental data of graphite}

The capacitance of graphite in contact with a $\mathrm{NaF}$ solution, shown in figure 9b, lacks a peak and its increase with absolute surface potential is slower than for silver. We calculate the capacitance again using the interfacial box model with steric effects, now including the additional carbon capacitance defined in eq 47, as we did for the graphite capacitance at the PZC in figure 8b. Because of the additional capacitance, the experimentally measured potential is not $\psi_{0}$, but $\psi_{0}+\psi_{\text {sub }}$, where $\psi_{\text {sub }}$ is the contribution from the carbon capacitance (figure 6). We calculate the potential difference across the carbon $\psi_{\text {sub }}$ by integrating eq 47

$$
\psi_{\mathrm{sub}}\left(\sigma_{0}\right)=\operatorname{sgn}\left(\sigma_{0}\right) \frac{z_{\mathrm{im}} \Sigma}{\varepsilon_{0}}\left(1-\mathrm{e}^{-\left|\sigma_{0}\right| / \Sigma}\right)
$$

We fit the capacitance curves by adjusting $\Sigma$ and using $a=0.24 \mathrm{~nm}$ for the effective diameter expected from the structure of the $\mathrm{NaF}$ ionic crystal, ${ }^{69}$ yielding $\Sigma=6 \mu \mathrm{C} / \mathrm{cm}^{2}$. All other parameters are the same as in figure 8b. In the Supporting Information, we explain the details of the fitting procedure and compare different ion diameters. The colored solid lines in figure $9 \mathrm{~b}$ show the results from the fitting procedure as a function of $\psi_{0}+\psi_{\text {sub }}$ for different salt concentrations, showing a quantitative agreement with the experimental data. At high voltages, the agreement with the experimental capacitance is better for higher salt concentrations. At concentrations above $10 \mathrm{mM}$, however, the theoretical capacitance curves collapse onto a single curve because the additional carbon capacitance, which is independent of salinity, dominates the total capacitance. Interestingly, however, previous theories on the 
graphite capacitance completely neglect the contribution of the diffuse-layer capacitance, which is important at low salinity, and only consider the case of high salinity. ${ }^{72,76,78}$ Therefore, our result is the first theoretical calculation for the finite-voltage capacitance of graphite with varying salt concentration.

Summarizing, the capacitance of silver at finite surface charge can be explained by the interfacial box model with steric effects. The effective ion diameter is $a=1.2 \mathrm{~nm}$, which is larger than those of $\mathrm{K}^{+}$and $\mathrm{PF}_{6}^{-}$found in ionic crystals. The capacitance of graphite can be explained by a $\sigma_{0}$-dependent capacitance of the substrate, eq 47 . The agreement with experimental data of graphite indicates strong nonelectrostatic attraction of charged species, $\alpha=-8.5$, the origin of which is yet unclear.
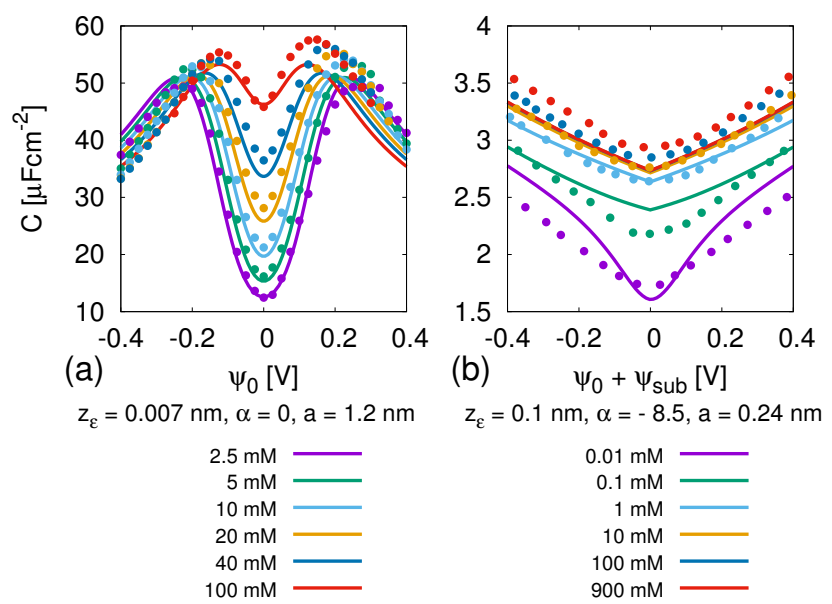

Figure 9: (a) Differential capacitance of a silver (110) surface in contact with a $\mathrm{KPF}_{6}$ solution as a function of the surface potential. The symbols are extracted from the continuous experimental curve. ${ }^{33}$ The experimental data shows a PZC at low salinity of $-0.975 \mathrm{~V}$ relative to the saturated calomel electrode $(\mathrm{SCE}),{ }^{33}$ which we have shifted to $\psi_{0}=0$ for clarity. The parameters used are $\varepsilon=78, T=298 \mathrm{~K}, z_{\varepsilon}=0.007 \mathrm{~nm}, \varepsilon_{\text {int }}=1, a=1.2 \mathrm{~nm}$, and $\alpha=0$. (b) Differential capacitance of stress-annealed pyrolytic graphite in contact with a $\mathrm{NaF}$ solution as a function of the total potential difference. The symbols are extracted from the experiment, ${ }^{37}$ where the voltage is measured relative to the normal hydrogen electrode (NHE),${ }^{37}$ so we have not shifted the potential. The parameters used are $\varepsilon=78, T=298 \mathrm{~K}$, $z_{\varepsilon}=0.1 \mathrm{~nm}, \varepsilon_{\text {int }}=1, a=0.24 \mathrm{~nm}, \alpha=-8.5, z_{\text {im }}=0.32 \mathrm{~nm}$, and $\Sigma=6 \mu \mathrm{C} / \mathrm{cm}^{2}$. 


\section{Electrokinetic power law}

The ion distribution determining the interfacial capacitance also affects the electro-osmotic velocity of the liquid when a tangential electric field is applied. In this section, we use the interfacial box model (figure 2bc) to model the electro-osmotic flow velocity, expressed in terms of the electrokinetic surface charge density. For an inhomogeneous viscosity profile, the hydrodynamics equation reads ${ }^{27,30}$

$$
\frac{d}{d z}\left[\eta_{\perp}(z) \frac{d u(z)}{d z}\right]+\rho(z) E_{\|}=0
$$

where $\eta_{\perp}(z)$ is the viscosity profile, $u(z)$ is the tangential velocity profile and $E_{\|}$is the applied tangential electric field. We define the zeta potential $\zeta$ by the solvent velocity infinitely far away from the surface $\left.u(z)\right|_{z \rightarrow \infty}$, which is the experimentally observed quantity $27,30,61$

$$
\zeta=-\frac{\left.\eta u\right|_{z \rightarrow \infty}}{\varepsilon \varepsilon_{0} E_{\|}}
$$

Combining eqs 1 and 49, and using the boundary conditions $d u(z) /\left.d z\right|_{z \rightarrow \infty}=0$ and $u(z=$ $0)=0$, the zeta potential follows as ${ }^{27,30,60}$

$$
\zeta=-\frac{\eta}{\varepsilon} \int_{0}^{\infty} \frac{\varepsilon_{\perp}(z)}{\eta_{\perp}(z)} \frac{d \psi}{d z} d z
$$

Consistent with the interfacial box model, we also approximate the viscosity profile by a box function,

$$
\eta_{\perp}(z)= \begin{cases}\eta_{\text {int }} & \text { for } 0<z<z_{\eta} \\ \eta & \text { for } z>z_{\eta},\end{cases}
$$

where $\eta_{\text {int }}$ is the viscosity in the interfacial layer. The viscosity profile can be obtained by MD simulations. ${ }^{26,27}$ For hydrophilic surfaces, the viscosity increases when approaching the interface, whereas for hydrophobic surfaces, there is a depletion layer, and the definition of a local viscosity is not straightforward. However, for both cases, it is easy to evaluate the 
slip length in a shear situation by extrapolating the bulk velocity gradient to the surface. Therefore, to determine $z_{\eta}$ and $\eta_{\text {int }}$, we define the slip length according to 26

$$
b=z_{\eta}\left(\frac{\eta}{\eta_{\text {int }}}-1\right)
$$

Similar to $\varepsilon_{\text {int }}$ and $\alpha_{ \pm}$, we assume $\eta_{\text {int }}$ and $z_{\eta}$ to remain constant when varying $\sigma_{0}$ and $c_{0}$, which means that we neglect the coupling of surface charge and slip, even though a finite coupling has been found in simulations. ${ }^{32}$ The width of the viscous interfacial layer generally differs from the dielectric one, ${ }^{27,30}$ depending on the assumed values of the interfacial dielectric constant $\varepsilon_{\text {int }}$ and viscosity $\eta_{\text {int }}$. For analytical tractability, however, we first examine the same width for both dielectric and viscosity profiles $\left(z_{\eta}=z_{\varepsilon}\right)$, allowing $\varepsilon_{\text {int }}$ and $\eta_{\text {int }}$ to vary. The case of a finite-viscosity layer with a width different from the dielectric layer is discussed later. From eqs 6, 51 and 52 the zeta potential follows as ${ }^{60,91}$

$$
\zeta=\gamma \psi_{0}+(1-\gamma) \psi^{*}
$$

where $\psi^{*}=\psi\left(z_{\varepsilon}\right)$, and where we define

$$
\gamma=\frac{\varepsilon_{\text {int }} \eta}{\varepsilon \eta_{\text {int }}}
$$

Based on the Grahame equation (eq 41), the electrokinetic surface charge density is defined $\operatorname{as}^{27,30}$

$$
\sigma_{\mathrm{ek}}=\sqrt{8 \varepsilon \varepsilon_{0} k_{\mathrm{B}} T c_{0}} \sinh \left(\frac{e \zeta}{2 k_{\mathrm{B}} T}\right) .
$$

For homogeneous systems, that means for $\eta_{\text {int }}=\eta$ and $\varepsilon_{\text {int }}=\varepsilon$, we obtain $\sigma_{\text {ek }}=\sigma_{0}$, i.e. the surface charge density estimated from electrokinetic experiments $\sigma_{\mathrm{ek}}$ equals the surface charge measured by titration $\sigma_{0}$. Therefore, deviations from $\sigma_{\mathrm{ek}}=\sigma_{0}$ are a measure for interfacial effects. ${ }^{27,30}$

We derive the asymptotic behavior of the electrokinetic surface charge density for high 
titrated surface charge density $\sigma_{0}$. Substituting eqs 36 and 37 into eq 54, and substituting eq 54 into eq 56 leads to ${ }^{60}$

$$
\left|\sigma_{\mathrm{ek}}\right| \approx \sqrt{\frac{\varepsilon \mathrm{e}^{\alpha_{\mp}}}{\varepsilon_{\mathrm{int}}}}\left(\frac{2 \varepsilon_{\mathrm{int}} \varepsilon_{0} k_{\mathrm{B}} T \operatorname{arccosh} \sqrt{\varepsilon \mathrm{e}^{\alpha_{\mp}} / \varepsilon_{\mathrm{int}}}}{e z_{\varepsilon} \sqrt{\varepsilon \mathrm{e}^{\alpha_{\mp}} / \varepsilon_{\mathrm{int}}-1}}\right)^{1-\gamma}\left|\sigma_{0}\right|^{\gamma} .
$$

Eq. (57) shows that the high- $\left|\sigma_{0}\right|$ behavior of $\left|\sigma_{\mathrm{ek}}\right|$, which has traditionally been interpreted as saturation, obeys a power law as a function of $\sigma_{0}$ instead. The exponent of the power law is analytically given by $\gamma=\varepsilon_{\text {int }} \eta / \varepsilon \eta_{\text {int }}$, which is the ratio of the bulk and interfacial viscous and dielectric properties. Furthermore, the prefactor of the power law in eq 57 is also given analytically. In contrast to the universal power laws found e.g. in phase transitions, the exponent and the prefactor of the electrokinetic power law are not universal, but depend on the interfacial material properties. Therefore, the interfacial parameters $\varepsilon_{\text {int }}, \eta_{\text {int }}, z_{\varepsilon}$ and $\alpha_{ \pm}$ can be extracted from the standard electrokinetic experiments via eq 57 .

\section{Simplified qualitative model using a uniform dielectric constant}

The surprising emergence of a power law can be easily explained qualitatively by the saturation of $\Psi^{*}$ and the inhomogeneity of the viscosity. As a minimal model, we consider the standard $\mathrm{PB}$ equation $\left(\varepsilon_{\text {int }}=\varepsilon\right.$ and $\alpha_{ \pm}=0$, see figure $\left.2 \mathrm{~d}\right)$. Then the surface and interfacial potentials, $\Psi_{0}$ and $\Psi^{*}$, become

$$
\begin{aligned}
& \Psi_{0}=2 \operatorname{arcsinh}\left(\frac{e \sigma_{0}}{\varepsilon \varepsilon_{0} k_{\mathrm{B}} T \kappa}\right) \\
& \Psi^{*}=2 \ln \frac{1+\mathrm{e}^{-\kappa z_{\varepsilon}} \tanh \left(\Psi_{0} / 4\right)}{1-\mathrm{e}^{-\kappa z_{\varepsilon}} \tanh \left(\Psi_{0} / 4\right)}
\end{aligned}
$$

and in the limit of $\sigma_{0} \rightarrow \pm \infty$,

$$
\begin{aligned}
& \Psi_{0} \approx \pm 2 \ln \frac{e \sigma_{0}}{\varepsilon \varepsilon_{0} k_{\mathrm{B}} T \kappa}, \\
& \Psi^{*} \approx \pm 2 \ln \frac{1+\mathrm{e}^{-\kappa z_{\varepsilon}}}{1-\mathrm{e}^{-\kappa z_{\varepsilon}}} .
\end{aligned}
$$


We substitute eqs 60 and 61 in eq 54, and take the limit of low salinity to arrive at

$$
\left|\sigma_{\mathrm{ek}}\right| \approx\left(\frac{2 \varepsilon \varepsilon_{0} k_{\mathrm{B}} T}{e z_{\varepsilon}}\right)^{1-\gamma}\left|\sigma_{0}\right|^{\gamma}
$$

where $\gamma=\eta / \eta_{\text {int }}$ is the ratio of the viscosities only. Eq. (62) can also be obtained by substituting $\alpha_{ \pm}=0$ and $\varepsilon_{\text {int }}=\varepsilon$ in eq 57 , so the simple derivation using the asymptotic eqs 60 and 61 is correct. This means that a power law is obtained even for the standard PB equation with uniform dielectric constant. When we consider a stagnant interfacial layer $\left(\eta_{\text {int }} \rightarrow \infty\right), \zeta$ is identical to $\psi^{*}$. In this limiting case, $\gamma=0$ and $\sigma_{\text {ek }}$ is completely saturated and equal to

$$
\sigma_{\mathrm{ek}}=\frac{2 \varepsilon \varepsilon_{0} k_{\mathrm{B}} T \kappa}{e \sinh \kappa z_{\varepsilon}}
$$

which is a decreasing function of salinity. Note that experimental values for $\sigma_{\mathrm{ek}}$ for hydrophilic surfaces indeed decrease with increasing salinity. ${ }^{3,6}$ In fact, the model with $\varepsilon_{\text {int }}=\varepsilon$, $\alpha=0$, and $\eta_{\text {int }} \rightarrow \infty$ has been studied by Lyklema and Overbeek, ${ }^{56}$ but it has not been used to explain the saturation of $\sigma_{\mathrm{ek}}$ because the model predicts much larger saturated zeta potentials than the experimentally measured ones. In summary, eq 62 provides an intuitive qualitative explanation for the electrokinetic saturation and the emergence of a power law, but quantitatively, this simplified model predicts relatively large values of $\eta_{\text {int }}$ because of the lack of the $\varepsilon_{\mathrm{int}} / \varepsilon$ factor in the exponent. Both the interfacial dielectric and viscosity effects are needed in order to explain the experimental data quantitatively.

\section{Comparison with experiments}

In this section, we compare our exact solution of $\sigma_{\text {ek }}$ given by eqs $21,25,54$, and 56 , as well as the asymptotic eq 57, with experiments. In typical experiments, the electrophoretic mobility of finite-sized particles is measured rather than the electro-osmotic mobility, so we compare our theory with electrophoresis measurements of colloidal solutions. For the colloids with finite radius, the relaxation or the double layer polarization effect is crucial to determine the 

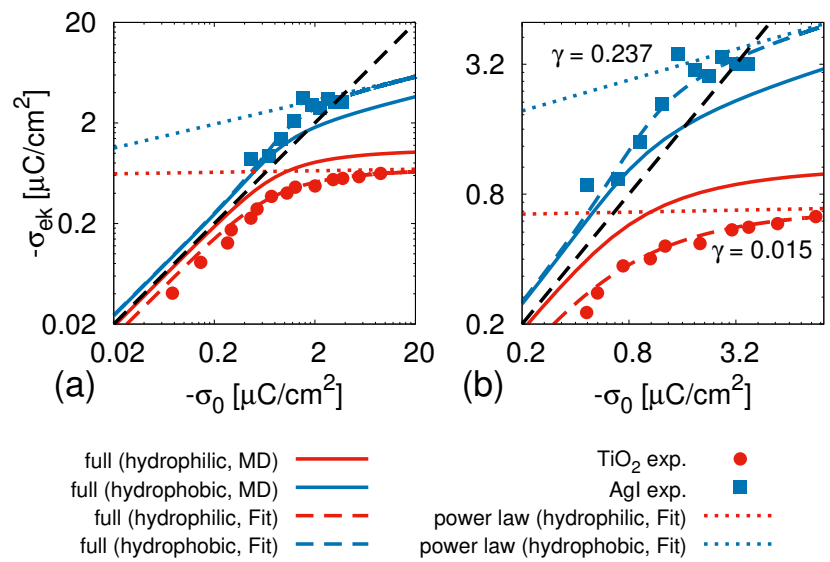

Figure 10: The electrokinetic surface charge density $\sigma_{\mathrm{ek}}$ for a $c_{0}=1 \mathrm{mM}$ electrolyte solution as a function of the titrated surface charge density $\sigma_{0}$ (double-logarithmic). The points are the experimental data extracted from refs 92 and 93. The colored lines show the full analytical solutions (eqs 21, 25, 54, and 56) with parameters obtained in MD simulations (solid lines, denoted MD) and with fitted parameters (broken lines, denoted Fit). The colored dotted lines denote eq 57 with fitted parameters. The black broken line denotes $\sigma_{\text {ek }}=\sigma_{0}$. We set $\varepsilon=78^{68}$ and $T=298 \mathrm{~K}$. The interfacial parameters are summarized in table 1 . (b) is a magnification of (a).

relation between the surface potential and the electrophoretic mobility. ${ }^{51,55}$ Therefore, we eliminate this effect from the experimental electrophoretic mobility data by the procedure described in detail in the Supporting Information, instead of using eq 50, which is only valid for planar surfaces or very large radii. Figure 10 shows a double-logarithmic plot of $\sigma_{\text {ek }}$. Without interfacial effects we obtain the black broken line $\sigma_{\mathrm{ek}}=\sigma_{0}$. The strong deviation of the experimental data from the black broken line reveals the importance of interfacial effects. The experimental data (symbols) have been obtained with $\mathrm{TiO}_{2}$ (rutile) and $\mathrm{AgI}$ in $\mathrm{KNO}_{3}$ aqueous solutions (the details of the $\sigma_{\mathrm{ek}}$ conversion are explained in the Supporting Information) ${ }^{92,93}$. At the hydrophobic surface, the data points $2 \mu \mathrm{C} / \mathrm{cm}^{2}<\left|\sigma_{\text {ek }}\right|<4 \mu \mathrm{C} / \mathrm{cm}^{2}$ have large uncertainties because they are close to the maximum point of the conversion function from the electrophoretic mobility to $\zeta$ (see Supporting Information). Note that hydrophobic surfaces are possibly affected by small amounts of impurities. ${ }^{94}$ The surface charge of $\mathrm{TiO}_{2}$ is governed by the $\mathrm{pH},{ }^{95}$ whereas the surface charge of AgI is governed by the bulk concentration of $\mathrm{Ag}^{+} \cdot{ }^{96-98}$ In general, $\mathrm{TiO}_{2}$ (rutile) is hydrophilic and $\mathrm{AgI}$ is 
hydrophobic. ${ }^{99-101}$ The colored solid and broken lines denote the model prediction defined by the exact solution of the interfacial box model without steric effects (eqs 21, 25, 54, and 56), and the colored dotted lines denote the asymptotic solution for large $\left|\sigma_{0}\right|$ (eq 57).

The interfacial parameters of the model are obtained by two different approaches. In the first approach, we take the parameters obtained from MD simulations of generic hydrophilic and hydrophobic surfaces, which have been independently verified by comparison with multiple sets of experimental data on surface capacitance, ${ }^{28,29}$ surface conduction and electrokinetics. ${ }^{27,30}$ Even though the simulations were performed for diamond surfaces instead of the surface types used in the experiments that we compare with, the advantage of this approach is the total absence of fit parameters. The specific adsorption parameter $\alpha$ is expected to depend sensitively on the combination of ions and surfaces used, but is set to $\alpha_{ \pm}=0$ due to the lack of literature data. For hydrophilic surfaces, the slip length $b$ and $\eta_{\text {int }}$ have been measured in MD simulations. ${ }^{26}$ Therefore, we use $z_{\varepsilon}=z_{\eta}=b\left(\eta / \eta_{\text {int }}-1\right)^{-1}$ as the width for both the dielectric and viscosity layers, and $\varepsilon_{\text {int }}$ is determined from the simulations by the value of the surface capacitance $\varepsilon_{\text {int }} / z_{\varepsilon} \cdot{ }^{27-30}$ For hydrophobic surfaces, slip is caused by a low-density depletion layer, which can be modeled by a slip boundary condition or by a low-viscosity layer. Either description constitutes an approximation that captures the large-scale hydrodynamic properties well, but at the molecular scale, both approximations are imperfect. For hydrophobic surfaces, we choose $\varepsilon_{\text {int }}=1$, fix $z_{\varepsilon}$ through the capacitance, and determine $\eta_{\text {int }}$ from the simulated value for $b$ by $\eta_{\text {int }}=\eta z_{\varepsilon} /\left(b+z_{\varepsilon}\right)$. For both surface types we observe a fair agreement between the experimental data and our analytical theory without fit parameters (figure 10, solid lines). However, especially for high $\left|\sigma_{0}\right|$ the amplitudes of the experimental data are different from the ones predicted by the theory without fit for both hydrophilic and hydrophobic surfaces (colored solid lines).

In the second approach, we extract the ion-surface interaction parameter $\alpha$ from a fit to the experimental data. At both surface types, excellent agreement is achieved by fitting only $\alpha$ (figure 10, red broken lines), resulting in the attractive interaction of $\alpha=-1.7$ 
for the hydrophilic surface, and the repulsive interaction of $\alpha=2.0$ for the hydrophobic surface. Other parameters are the same as those extracted from MD simulations and are given in table 1. Details of the fitting procedure are given in the Supporting Information. The stronger repulsion of ions from the interfacial layer at hydrophobic surfaces $(\alpha=2.0)$ than at hydrophilic surfaces $(\alpha=-1.7)$ is qualitatively consistent with ionic potentials of mean force calculated by MD simulations of hydrophobic and hydrophilic self-assembled monolayers. ${ }^{27,62}$ The fitted and MD parameters used in figure 10 are summarized in table 1.

In summary, our theory and the comparison with experimental data reveal that the electrokinetic surface charge $\sigma_{\text {ek }}$ exhibits a power law behavior with a small but non-zero exponent ( $\gamma=0.237$ for the hydrophobic and $\gamma=0.015$ for the hydrophilic surface), determined by the subnanometer-wide interfacial structure. Therefore, if accurate experimental data for highly charged surfaces are available, eq 57 provides a method to measure the parameters characterizing the interfacial structure, and in particular the elusive interfacial viscosity, by standard electrokinetic experiments.

Table 1: Interfacial parameters from molecular dynamics simulations (MD) ${ }^{26,28}$ and from fitting (Fit). The interfacial capacitance $\varepsilon_{\text {int }} / z_{\varepsilon}$ and the slip length $b$ are shown for comparing the difference between MD and Fit.

\begin{tabular}{cccccccc}
\hline & & $\varepsilon_{\text {int }}$ & $\begin{array}{c}z_{\varepsilon} \\
(\mathrm{nm})\end{array}$ & $\eta_{\text {int }} / \eta$ & $\alpha$ & $\begin{array}{c}\varepsilon_{\text {int }} / z_{\varepsilon} \\
\left(\mathrm{nm}^{-1}\right)\end{array}$ & $\begin{array}{c}b \\
(\mathrm{~nm})\end{array}$ \\
\hline hydrophilic & MD & 4.4 & 0.44 & $3.7^{26}$ & 0.0 & $10^{28}$ & $-0.32^{26}$ \\
$\left(\mathrm{TiO}_{2}\right)$ & Fit & 4.4 & 0.44 & 3.7 & -1.7 & 10 & -0.32 \\
\hline hydrophobic & MD & 1.0 & 0.12 & 0.054 & 0.0 & $8.3^{28}$ & $2.2^{26}$ \\
$(\mathrm{AgI})$ & Fit & 1.0 & 0.12 & 0.054 & 2.0 & 8.3 & 2.1 \\
\hline
\end{tabular}

\section{Different widths of the interfacial viscosity and dielectric layers}

Now we examine the more general case where $z_{\eta} \neq z_{\varepsilon}$. Note that this more general model contains both the model used previously in this paper but also the pure slip boundary condition $\left(z_{\eta} \rightarrow 0\right)$ as limiting cases. We calculate $\sigma_{\text {ek }}$ for various values of $z_{\eta} / z_{\varepsilon}$ while 
keeping the slip length fixed at $b=z_{\eta}\left(\eta / \eta_{\text {int }}-1\right)$. The zeta potential follows from eq 51 as

$$
\zeta=\gamma \psi_{0}+\left(\gamma^{\prime}-\gamma\right) \psi_{1}+\left(1-\gamma^{\prime}\right) \psi_{2}
$$

where $\gamma^{\prime}, \psi_{1}$, and $\psi_{2}$ are given by

$$
\begin{aligned}
& \gamma^{\prime}=\varepsilon_{\text {int }} / \varepsilon, \quad \psi_{1}=\psi\left(z_{\eta}\right), \quad \psi_{2}=\psi\left(z_{\varepsilon}\right), \quad \text { for } \quad z_{\eta}<z_{\varepsilon}, \\
& \gamma^{\prime}=\eta / \eta_{\text {int }}, \quad \psi_{1}=\psi\left(z_{\varepsilon}\right), \quad \psi_{2}=\psi\left(z_{\eta}\right), \quad \text { for } \quad z_{\eta}>z_{\varepsilon} .
\end{aligned}
$$

Note that the exact solution of the $\mathrm{PB}$ equation does not change when we set $z_{\eta} \neq z_{\varepsilon}$. However, $\zeta$ and its dependence on $\gamma$ change because the values of $\psi_{1}$ and $\psi_{2}$ depend on $z_{\eta}$. In the case of $z_{\eta}>z_{\varepsilon}, \psi_{1}$ and $\psi_{2}$ are saturated in the limit of high surface charge density, and therefore a power law $\left|\sigma_{\text {ek }}\right| \sim\left|\sigma_{0}\right|^{\gamma}$ holds, similar to the $z_{\eta}=z_{\varepsilon}$ case. In contrast, $\psi_{1}$ is not saturated in the high surface charge limit in the case of $z_{\eta}<z_{\varepsilon}$, and $\sigma_{\text {ek }}$ no longer follows the power law. The limit $z_{\eta} \rightarrow 0$ corresponds to the usual pure slip boundary condition,

$$
\left.b \frac{d u}{d z}\right|_{z=0}=u(0)
$$

and the zeta potential is equal to

$$
\zeta=\gamma^{\prime} \psi_{0}+\left(1-\gamma^{\prime}\right) \psi^{*}+\frac{b \sigma_{0}}{\varepsilon \varepsilon_{0}}
$$

where $\gamma^{\prime}=\varepsilon_{\text {int }} / \varepsilon$ is the ratio of the dielectric constants only. Eq. (68) shows that a positive slip length enhances the zeta potential whereas a negative slip length decreases it, in agreement with previous results. ${ }^{61}$ Note that eq 67 is typically used with a positive $b$ for hydrophobic surfaces, but not for hydrophilic surfaces, where $b$ is negative. We call eq 67 the 
pure slip boundary condition even for hydrophilic surfaces, though it is "physically no-slip".

Figure 11 shows $\sigma_{\text {ek }}$ as a function of $\sigma_{0}$ for varying $z_{\eta} / z_{\varepsilon}$ while keeping the slip length $b$ fixed. All the parameters used are the same as the fitted ones in table 1 . The reason for varying $z_{\eta}$ while fixing $b$ and $\varepsilon_{\text {int }} / z_{\varepsilon}$ in our comparison (figure 11 ) is that $b$ and $\varepsilon_{\text {int }} / z_{\varepsilon}$ are measurable by macroscopic experiments, whereas measuring $z_{\eta}$ is difficult. Even for the case of $z_{\eta}=10 z_{\varepsilon}$, curves of $\sigma_{\mathrm{ek}}$ as a function of $\sigma_{0}$ are similar to the case of $z_{\eta}=z_{\varepsilon}$ for both hydrophilic and hydrophobic surfaces. However, for $z_{\eta}=0.1 z_{\varepsilon}$, the change from the results of $z_{\eta}=z_{\varepsilon}$, and thus the deviation from the experimental data, is large. In the case of $z_{\eta} \rightarrow 0$, the hydrophilic $\sigma_{\text {ek }}$ curve (red dotted-dashed line) is lower than the result of $z_{\eta}=z_{\varepsilon}$, whereas the hydrophobic curve (blue dotted-dashed line) is higher, in agreement with the findings of eq 68. We conclude that the results produced by a pure-slip description, which is equivalent to choosing a vanishing thickness of the viscosity layer $z_{\eta}$, in combination with an interfacial dielectric layer are in strong disagreement with the experimental evidence. However, a good match with experimental data is obtained when the value of the viscosity dividing surface position $z_{\eta}$ is of the order of the dielectric width $z_{\varepsilon}$.
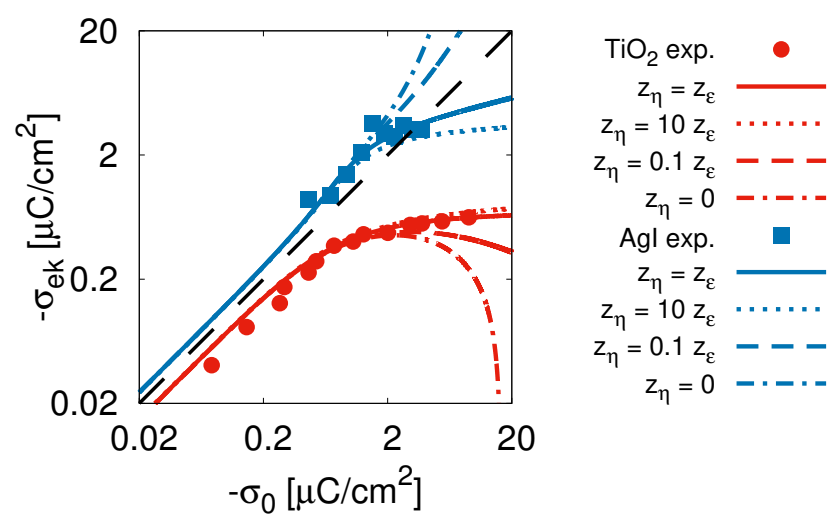

Figure 11: Electrokinetic surface charge density $\sigma_{\mathrm{ek}}$ for varying viscous dividing surface positions $z_{\eta}$ while keeping the slip length fixed. Other parameters are fitted and described in table 1 and the salt concentration is $1 \mathrm{mM}$. The black broken line denotes $\sigma_{\mathrm{ek}}=\sigma_{0}$. 


\section{Steric effects}

Finally, we examine the effect of steric interactions on the electrokinetic power law. It has been shown previously that the electrophoretic mobility is strongly enhanced when steric effects are dominant. ${ }^{48}$ Figure 12 shows $\sigma_{\text {ek }}$ as a function of $\sigma_{0}$ with steric effects included. We use $a=0.3 \mathrm{~nm}$ which corresponds to the ionic diameter of potassium. ${ }^{69}$ All the other parameters used in figure 12 are the same as the fitted parameters in table 1 . The steric effect significantly enhances $\sigma_{\text {ek }}$ in the range of $\left|\sigma_{0}\right| \geq 20 \mu \mathrm{C} / \mathrm{cm}^{2}$. However, there is still a wide range of $2-20 \mu \mathrm{C} / \mathrm{cm}^{2}$ in which the asymptotic power law is valid. Moreover, the surface charge density in colloidal systems is typically the result of the dissociation of surface groups, and has an upper bound given by the surface density of dissociable groups of the order of $\left|\sigma_{0}\right|=10 \mu \mathrm{C} / \mathrm{cm}^{2}$. Therefore, the high surface charges where steric effects enhance $\sigma_{\text {ek }}$ are typically only reached with specially designed ions.

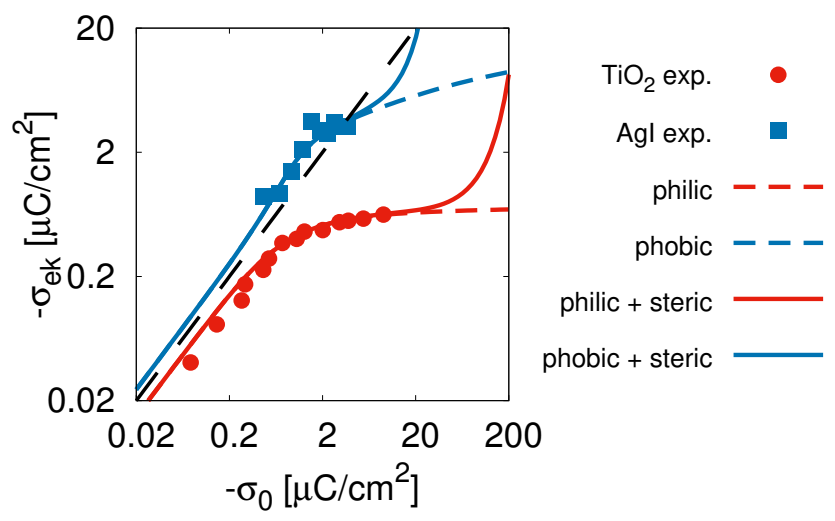

Figure 12: The electrokinetic surface charge density $\sigma_{\mathrm{ek}}$ for hydrophilic and hydrophobic surfaces in contact with a $1 \mathrm{mM}$ electrolyte solution, including steric effects (solid lines) based on eqs 1, 2, 4, 54, and 56, compared with the case without steric effects (broken lines) based on eqs 21, 25, 54, and 56. The effective diameter of the ions is $a=0.3 \mathrm{~nm}$. Other parameters are the fitted ones described in table 1. The black broken line denotes $\sigma_{\text {ek }}=\sigma_{0}$.

\section{Conclusions}

The modified dielectric constant, viscosity and ion adsorption potential in the subnanometerwide interfacial layer dominate the surface capacitance and the electrokinetic properties of 
aqueous solutes, leading to deviations from the standard PB and Stokes equations. Even though the underlying molecular interactions are complex and involve a multitude of specific parameters, we show that the effects of the interfacial layer can be accurately and effectively modeled by an interfacial box model. The primary advantage of our interfacial box model is that the exact solution is available (in the case without steric effects) enabling the analysis of experimental data by simple fitting procedures, thus giving direct access to the effective properties of the subnanometer-wide interfacial layer. Moreover, the model is general and flexible enough to analyze a multitude of phenomena originating in the electric double layer within the same framework. For the simultaneous extraction of all parameters of the interfacial box model, however, concurrent measurements of the capacitance and the electro-osmotic mobility for identical surface types are important. ${ }^{102}$

We apply our interfacial box model to different sets of literature experimental data. First, we show that the capacitance of a silver (110) surface in contact with a $\mathrm{KPF}_{6}$ solution agrees with the result of the interfacial box model, giving a dielectric interfacial layer width of $z_{\varepsilon}=0.007 \mathrm{~nm}$. We attribute this narrow width of the interfacial layer compared to the width found in classical MD simulations $\left(z_{\varepsilon}=0.1 \mathrm{~nm}\right)$ to the spill-over of electrons from the metal. Fitting the interfacial box model to the capacitance of planar, non-porous pyrolytic graphites in contact with $\mathrm{NaF}$ solutions shows the presence of strongly adsorbing charged species $(\alpha=-8.5)$. MD simulations of simple ions on different types of surfaces show that their adsorption potentials are typically smaller, ${ }^{27}$ suggesting that the adsorbing species in the capacitance measurements are, for example, charged organic surfactants instead. ${ }^{88,89}$ For pyrolytic graphites, the capacitance needs an additional carbon capacitance of $C_{\text {sub }}=$ $2.8 \mu \mathrm{F} / \mathrm{cm}^{2}$ in quantitative agreement with density functional calculations. ${ }^{78}$ This substrate capacitance corresponds to a shift of the image plane of $z_{\mathrm{im}}=0.32 \mathrm{~nm}$ toward the solid phase, which can be rationalized by the formation of a diffuse-layer like structure of the surface charge on the carbon. The calculated differential capacitance at finite potentials is also in good agreement with the experimental data for both the silver and graphite surfaces. 
Second, we derive that the electro-osmotic flow velocity, quantified by the electrokinetic surface charge density, obeys a power law with increasing surface charge density, with an exponent equal to $\varepsilon_{\text {int }} \eta / \varepsilon \eta_{\text {int }}$. We examine the robustness of this power law by using different widths for the interfacial dielectric and viscosity layers and by including steric ion-ion interactions. For both hydrophilic and hydrophobic surfaces, we show that a model with a finite-viscosity layer describes the experimental data better than a model with a pure slip boundary condition, which corresponds to the limit of a vanishing interfacial viscosity layer. Steric interactions enhance the electro-osmosis, eventually obscuring the power law at high charge density, but for medium-sized ions the onset of the steric effects starts beyond the experimentally accessible surface charge density.

The success of our analytically solvable model at capturing a wide range of experimental results provides a promising route to extract the effective interfacial properties of aqueous solutes from standard electrostatic and electrokinetic experiments, and to relate the results to a molecular model of the interface. Although complete experimental surveys of electrostatic and electrokinetic properties on a single surface type are lacking at present, recent electrokinetic experiments using carbon and boron-nitride nanotubes ${ }^{103-105}$ provide an exciting new area for the future application of our theoretical framework.

\section{Acknowledgement}

This work was supported by Grant-in-Aid for JSPS Research Fellow Number 16J00042 and funding from the Deutsche Forschungsgemeinschaft (project NE 810/10-1).

\section{Supporting Information}

Derivation of the analytic solution of extended Poisson-Boltzmann equation, capacitance data, discussion of capacitance, conversion methods of the electrokinetic surface charge density, and fitting procedures of the ion-surface interaction parameters in the electrokinetic 
power law.

\section{Author biography}

\section{Yuki Uematsu:}

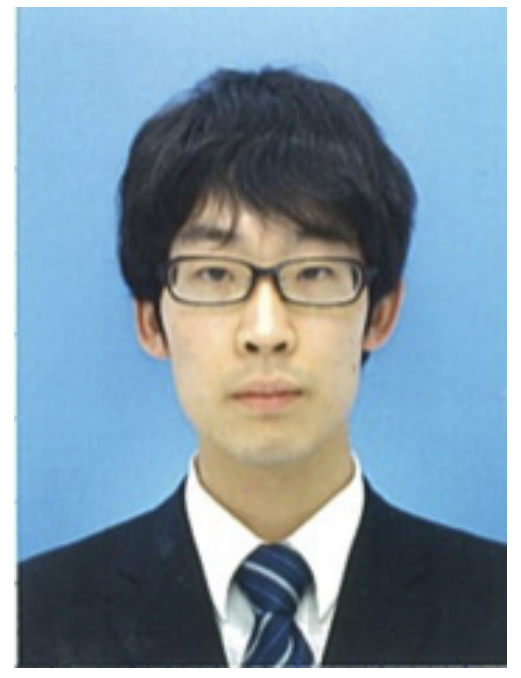

Yuki Uematsu studied physics at Kyoto University and received his Ph.D. in theoretical soft matter physics, especially electrokinetics of polymer solutions. Since 2016, he has a JSPS Research Fellowship and spent his first postdoc position at the Free University of Berlin. Currently, he holds a postdoc position at the department of chemistry, Kyushu University.

\section{Roland R. Netz:}

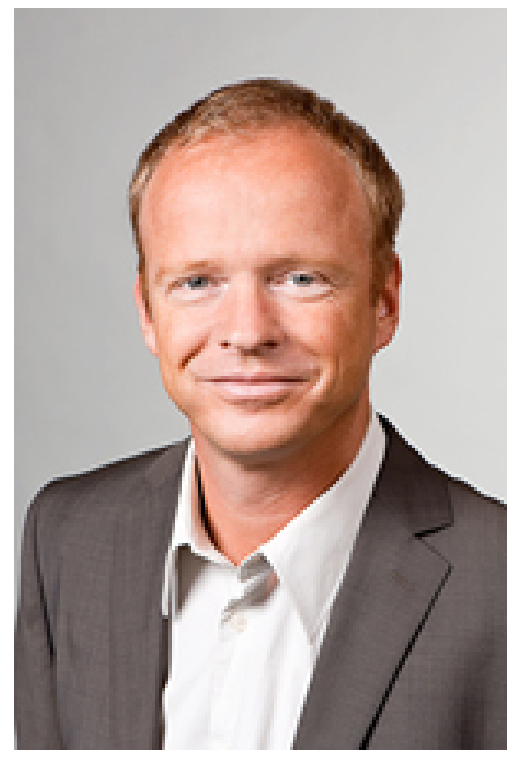


Roland R. Netz studied physics at the Technical University of Berlin and at MIT and received his Ph.D. in 1994 from the University of Cologne. After postdocs at Tel-Aviv University, UCSB, Seattle, Institute Charles Sadron in Strasbourg, and CEA in Paris, he was a research associate at the Max-Planck Institute for Colloids and Interfaces in Potsdam. He was appointed associate professor of physics at the LMU Munich in 2002 and full professor of physics at the TU Munich in 2004. Since 2011, he has held a chair in theoretical bio-soft matter physics at the Free University of Berlin.

\section{Douwe Jan Bonthuis:}

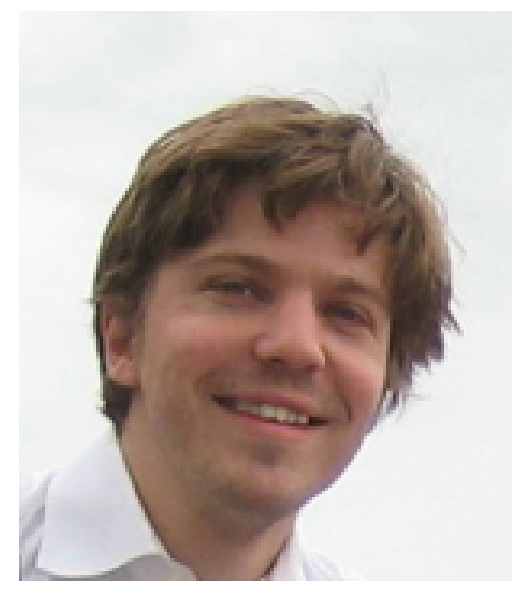

Douwe Jan Bonthuis studied physics at Delft University of Technology and Harvard University. He received his Ph.D. in theoretical physics in 2012 from the Technical University of Munich. From 2012, he held a position as a Glasstone Fellow at the Rudolf Peierls Centre for Theoretical Physics, University of Oxford. Since 2016, he works at the Free University of Berlin.

\section{References}

(1) Dukhin, S. S.; Derjaguin, B. V. In Surafce and Colloid Science; Matijrvic, E., Ed.; Wiley: New York, 1974; Vol. 7.

(2) Russel, W. B.; Saville, D. A.; Schowalter, W. R. Colloidal Dispersions; Cambridge University Press: Cambridge, 1989. 
(3) Lyklema, J. Fundamentals of Interface and Colloid Science; Academic Press: London, 1995; Vol. 3.

(4) Stern, O. Zur Theorie der Elektrolytischen Doppelschicht. Zeitschrift für Elektrochemie und Angewandte Physikalische Chemie 1924, 30, 508-516.

(5) Grahame, D. C. The Electrical Double Layer and the Theory of Electrocapillarity. Chem. Rev. 1947, 41, 441-501.

(6) Lyklema, J. On the slip process in electrokinetics. Colloid Surf. A 1994, 92, 41-49.

(7) Lyklema, J.; Rovillard, S.; Coninck, J. D. Electrokinetics: The Properties of the Stagnant Layer Unraveled. Langmuir 1998, 14, 5659-5663.

(8) Kunz, W. Specific ion effects in colloidal and biological systems. Curr. Opin. Colloid Interface Sci. 2010, 15, 34-39.

(9) Horinek, D.; Serr, A.; Bonthuis, D. J.; Boström, M.; Kunz, W.; Netz, R. R. Molecular Hydrophobic Attraction and Ion-Specific Effects Studied by Molecular Dynamics. Langmuir 2008, 24, 1271-1283.

(10) Bazant, M. Z.; Storey, B. D.; Kornyshev, A. A. Double Layer in Ionic Liquids: Overscreening versus Crowding. Phys. Rev. Lett. 2011, 106, 046102.

(11) Marčelja, S.; Radić, N. Repulsion of interfaces due to boundary water. Chem. Phys. Lett. 1976, 42, 129.

(12) Israelachvili, J.; Wennerström, H. Entropic forces between amphiphilic surfaces in liquids. J. Phys. Chem. 1992, 96, 520.

(13) Booth, F. The Dielectric Constant of Water and the Saturation Effect. J. Chem. Phys. $\mathbf{1 9 5 1 , 1 9 , 3 9 1 . ~}$ 
(14) Grahame, D. C. Diffuse Double Layer Theory for Electrolytes of Unsymmetrical Valence Types. J. Chem. Phys. 1953, 21, 1054.

(15) Abrashkin, A.; Andelman, D.; Orland, H. Dipolar Poisson-Boltzmann Equation: Ions and Dipoles Close to Charge Interfaces. Phys. Rev. Lett. 2007, 99, 077801.

(16) Bikerman, J. J. Structure and capacity of electrical double layer. Philos. Mag. 7 1942, 33, 384-397.

(17) Eigen, M.; Wicke, E. The Thermodynamics of Electrolytes at Higher Concentration. J. Phys. Chem. 1954, 58, 702.

(18) Kralj-Iglič, V.; Iglič, A. Influence of finite size of ions on elecrostatic properties of electric double layer. Electrotech. Rev. Ljubljana, Slovenija 1994, 61, 127-133.

(19) Borukhov, L.; Andelman, D.; Orland, H. Adsorption of large ions from an electrolyte solution: a modified PoissonBoltzmann equation. Electrochim. Acta 2000, 46, 221.

(20) Carnahan, N. F.; Starling, K. E. Equation of State for Nonattracting Rigid Spheres. J. Chem. Phys. 1969, 51, 635-636.

(21) Biesheuvel, P. M.; van Soestbergen, M. Counterion volume effects in mixed electrical double layers. J. Colloid Int. Sci. 2007, 316, 490-499.

(22) Stout, R. F.; Khair, A. S. Influence of ion sterics on diffusiophoresis and electrophoresis in concentrated electrolytes. Phys. Rev. Fluids 2017, 2, 014201.

(23) Boström, M.; Williams, D. R. M.; Ninham, B. W. Surface Tension of Electrolytes: Specific Ion Effects Explained by Dispersion Forces. Langmuir 2001, 17, 4475-4478.

(24) Horinek, D.; Netz, R. R. Specific ion adsorption at hydrophobic solid surfaces. Phys. Rev. Lett. 2007, 99, 226104. 
(25) Jungwirth, P.; Tobias, D. J. Specific ion effects at the air/water interface. Chem. Rev. 2006, 106, 1259-1281.

(26) Sendner, C.; Horinek, D.; Bocquet, L.; Netz, R. R. Interfacial water at hydrophobic and hydrophilic surfaces: slip, viscosity, and diffusion. Langmuir 2009, 25, 1076810781.

(27) Bonthuis, D. J.; Netz, R. R. Beyond the Continuum: How Molecular Solvent Structure Affects Electrostatics and Hydrodynamics at SolidElectrolyte Interfaces. J. Phys. Chem. B 2013, 117, 11397-11413.

(28) Bonthuis, D. J.; Gekle, S.; Netz, R. R. Dielectric Profile of Interfacial Water and its Effect on Double-Layer Capacitance. Phys. Rev. Lett. 2011, 10\%, 166102.

(29) Bonthuis, D. J.; Gekle, S.; Netz, R. R. Profile of the Static Permittivity Tensor of Water at Interfaces: Consequences for Capacitance, Hydration Interaction and Ion Adsorption. Langmuir 2012, 28, 7679.

(30) Bonthuis, D. J.; Netz, R. R. Unraveling the Combined Effects of Dielectric and Viscosity Profiles on Surface Capacitance, Electro-Osmotic Mobility, and Electric Surface Conductivity. Langmuir 2012, 28, 16049-16059.

(31) Schlaich, A.; Knapp, E. W.; Netz, R. R. Water Dielectric Effects in Planar Confinement. Phys. Rev. Lett. 2016, 117, 048001.

(32) Joly, L.; Ybert, C.; Trizac, E.; Bocquet, L. Liquid friction on charged surfaces: From hydrodynamic slippage to electrokinetics. J. Chem. Phys. 2006, 125, 204716.

(33) Valette, G. Double layer on silver single-crystal electrodes in contact with electrolytes having anions which present a slight specific adsorption: Part I. The (110) face. J. Electroanal. Chem. 1981, 122, 285-297. 
(34) Valette, G. Double layer on silver single crystal electrodes in contact with electrolytes having anions which are slightly specifically adsorbed: Part II. The (100) face. J. Electroanal. Chem. 1982, 138, 37-54.

(35) Persons, R.; Zobel, F. G. R. The interphase between mercury and aqueous sodium dihydrogen phosphate. J. Electroanal. Chem. 1965, 9, 333-348.

(36) Randin, J.-P.; Yeager, E. Differential Capacitance Study of Stres-Annealed Pyrolytic Graphite Electrodes. J. Electrochem. Soc. 1971, 118, 711-714.

(37) Randin, J.-P.; Yeager, E. Differential capacitance study on the basal plane of stressannealed pyrolytic graphite. J. Electroanal. Chem. 1972, 36, 257-276.

(38) Ji, H.; Zhao, X.; Qiao, Z.; Jung, J.; Zhu, Y.; Lu, Y.; Zhang, L. L.; MacDonald, A. H.; Ruoff, R. S. Capacitance of carbon-based electrical double-layer capacitors. Nature Comm. 2014, 5, 3317 .

(39) Langmuir, I. The adsorption of gases on plane surfaces of glass, mica and platinum. J. Am. Chem. Soc. 1918, 40, 1361-1403.

(40) Trefalt, G.; Behrens, S. H.; Borkovec, M. Charge regulation in the electrical double layer: ion adsorption and surface interactions. Langmuir 2016, 32, 380-400.

(41) Hughes, C.; Yeh, L. H.; Qian, A. Field Effect Modulation of Surface Charge Property and Electroosmotic Flow in a Nanochannel: Stern Layer Effect. J. Phys. Chem. C 2013, 117, 9322-9331.

(42) Mei, L.; Chou, T. H.; Cheng, Y. S.; Huang, M. J.; Yeh, L. H.; Qian, S. Electrophoresis of pH-regulated nanoparticles: impact of the Stern layer. Phys. Chem. Chem. Phys. 2016, 18, 9927-9934.

(43) Borukhov, L.; Andelman, D.; Orland, H. Steric Effects in Electrolytes: A Modified Poisson-Boltzmann Equation. Phys. Rev. Lett. 1997, 79, 435. 
(44) Kilic, M. S.; Bazant, M. Z.; Ajdari, A. Steric effects in the dynamics of electrolytes at large applied voltages. I. Double-layer charging. Phys. Rev. E 2007, 75, 021502.

(45) Bazant, M.; Kilic, M. S.; Storey, B. D.; Ajdari, A. Towards an understanding of induced-charge electrokinetics at large applied voltages in concentrated solutions. Adv. Colloid Int. Sci. 2009, 152, 48-88.

(46) Hatlo, M. M.; van Roij, R.; Lue, L. The electric double layer at high surface potentials: The influence of excess ion polarizability. EPL 2012, 97, 28010.

(47) Nakayama, Y.; Andelman, D. Differential capacitance of the electric double layer: the interplay between ion finite size and dielectric decrement. J. Chem. Phys. 2015, 142, 044706.

(48) Khair, A. S.; Squires, T. M. Ion steric effects on electrophoresis of a colloidal particle. J. Fluid Mech. 2009, 640, 343-356.

(49) Landstorfer, M.; Guhlke, C.; Dreyer, W. Theory and structure of the metal-electrolyte interface incorporating adsorption and solvation effects. Electrochim. Acta 2016, 201, $187-219$.

(50) López-García, J. J.; Horno, J.; Grosse, C. Ion size effects on the dielectric and electrokinetic properties in aqueous colloidal suspensions. Curr. Opin. Colloid Int. Sci. 2016, 24, 23-31.

(51) O'Brien, L. R.; White, L. R. Electrophoretic mobility of a spherical colloidal particle. J. Chem. Soc., Faraday Trans. 2 1978, 74, 1607-1626.

(52) von Smoluchowski, M. Versuch einer mathematischen Theorie der Kiagulationskinetik kolloider Lösungen. Z. Phys. Chem. 1918, 92, 129-168.

(53) Hückel, E. Die kataphorese der kugel. Physik. Z. 1924, 25, 204. 
(54) Henry, D. C. The Cataphoresis of Suspended Particles. Part I. The Equation of Cataphoresis. Proc. R. Soc. London, Ser. A 1931, 133, 106.

(55) Wiersema, P. H.; Loeb, A. L.; Overbeek, J. T. G. Calculation of the electrophoretic mobility of a spherical colloid particle. J. Colloid Interface Sci. 1966, 22, 78-99.

(56) Lyklema, J.; Overbeek, J. T. G. On the interpretation of electrokinetic potentials. J. Colloid Sci. 1961, 16, 501-512.

(57) Attard, P.; Antelmi, D.; Larson, I. Comparison of the Zeta Potential with the Diffuse Layer Potential from Charge Titration. Langmuir 2000, 16, 1542-1552.

(58) Borkovec, M.; Behrens, S. H.; Semmler, M. Observation of the Mobility Maximum Predicted by the Standard Electrokinetic Model for Highly Charged Amidine Latex Particles. Langmuir 2000, 16, 5209-5212.

(59) Yeh, I.-C.; Berkowitz, M. L. Dielectric constant of water at high electric fields: Molecular dynamics study. J. Chem. Phys. 1999, 110, 7935.

(60) Uematsu, Y.; Netz, R. R.; Bonthuis, D. J. Power-law electrokinetic behavior as a direct probe of effective surface viscosity. Chem. Phys. Lett. 2017, 670, 11-15.

(61) Bonthuis, D. J.; Uematsu, Y.; Netz, R. R. Interfacial layer effects on surface capacitances and electro-osmosis in electrolytes. Phil. Trans. R. Soc. A 2016, 374, 20150033.

(62) Schwierz, N.; Horinek, D.; Netz, R. R. Reversed anionic Hofmeister series: the interplay of surface charge and surface polarity. Langmuir 2010, 26, 7370-7379.

(63) Rinne, K.; Gekle, S.; Netz, R. R. Ion-Specific Solvation Water Dynamics: Single Water versus Collective Water Effects. J. Phys. Chem. A 2014, 118, 11667-11677.

(64) Rinne, K.; Gekle, S.; Netz, R. R. Impact of secondary structure and hydration water on the dielectric spectrum of poly-alanine and possible relation to the debate on slaved versus slaving water. J. Chem. Phys. 2014, 141, 214502. 
(65) Abramowitz, M.; Stegun, I. A. Handbook of Mathematical Functions, 9th ed.; Dover Publications: New York, 1972.

(66) Verwey, E.; Overbeek, J. Theory of the Stability of Lyophobic Colloids; Elsevier: New York, 1948.

(67) Behrens, S. H.; Borkovec, M. Exact Poisson-Boltzmann solution for the interaction of dissimilar charge-regulating surfaces. Phys. Rev. E 1999, 60, 7040-7048.

(68) Owen, B. B.; Miller, R. C.; Milner, C. E.; Cogan, H. L. The dielectric constant of water as a function of temperature and pressure. J. Phys. Chem. 1961, 65, 2065.

(69) Shannon, R. D. Revised effective ionic radii and systematic studies of interatomic distances in halides and chalcogenides. Acta Cryst. A 1976, 32, 751-767.

(70) Gouy, M. Sur la constitution de la charge électrique à la surface d'un électrolyte. $J$. Phys. Theor. Appl. 1910, 9, 457-468.

(71) Chapman, D. L. A contribution to the theory of electrocapillarity. Phil. Mag. 6 1913, $25,475-481$.

(72) Gerischer, H.; McIntyre, R.; Scherosn, D.; Storck, W. Density of the electronic states of graphite: derivation from differential capacitance measurements. J. Phys. Chem. 1987, 91, 1930-1935.

(73) Schmickler, W. Electronic Effects in the Electric Double Layer. Chem. Rev. 1996, 96, $3177-3200$.

(74) Schmickler, W.; Henderson, D. The interphase between jellium and a hard sphere electrolyte. A model for the electric double layer. J. Chem. Phys. 1984, 80, 33813386. 
(75) Schmickler, W.; Henderson, D. The interphase between jellium and a hard sphere electrolyte: Capacity-charge characteristics and dipole potentials. J. Chem. Phys. 1986, $85,1650-1657$.

(76) Gerischer, H. An interpretation of the double layer capacity of graphite electrodes in relation to the density of states at the Fermi level. J. Phys. Chem. 1985, 89, 42494251.

(77) Federov, M. V.; Kornyshev, A. A. Ionic Liquids at Electrified Interfaces. Chem. Rev. 2014, 114, 2978-3036.

(78) Luque, N. B.; Schmickler, W. The electric double layer on graphite. Electrochim. Acta 2012, $71,82-85$.

(79) Uematsu, Y.; Netz, R. R.; Bonthuis, D. J. The effects of ion adsorption on the potential of zero charge and the differential capacitance of charged aqueous interfaces. J. Phys.: Condens. Matter 2018, 30, 064002.

(80) Daikhin, L. I.; Kornyshev, A. A.; Urbakh, M. Double-layer capacitance on a rough metal surface. Phys. Rev. E. 1996, 53, 6192-6199.

(81) López, M. J.; Cabria, I.; Alonso, J. A. Simulated porosity and electronic structure of nanoporous carbons. J. Chem. Phys. 2011, 135, 104706.

(82) Baskin, A.; Král, P. Electronic structures of porous nanocarbons. Sci. Rep. 2011, 36, $1-7$.

(83) Carnie, S. L.; Chan, D. Y. C. The structure of electrolytes at charged surfaces: Iondipole mixtures. J. Chem. Phys. 1980, 73, 2949-2957.

(84) Blum, L.; Henderson, D. Mixtures of hard ions and dipoles against a charged wall: The Ornstein-Zernike equation, some exact results, and the mean spherical approximation. J. Chem. Phys. 1981, 74, 1902-1910. 
(85) Lang, N. D.; Kohn, W. Theory of Metal Surfaces: Induced Surface Charge and Image Potential. Phys. Rev. B. 1973, 7, 3541-3550.

(86) Xia, J.; Chen, F.; Li, J.; Tao, N. Measurement of the quantum capacitance of graphene. Nature Nanotech. 2009, 4, 505-509.

(87) Sharma, P. Capacitance of graphene in aqueous electrolytes: The effects of dielectric saturation of water and finite size of ions. Phys. Rev. B. 2014, 90, 125415.

(88) Warszyński, P.; Barzyk, W.; Lunkenheimer, K.; Fruhner, H. Surface Tension and Surface Potential of Na n-Dodecyl Sulfate at the Air-Solution Interface: Model and Experiment. J. Phys. Chem. B 1998, 102, 10948-10957.

(89) Ozeki, S.; Ikeda, S. The adsorption of dodecyldimethylammonim chloride on aqueous surfaces of concentrated $\mathrm{NaCl}$ solutions. Bull. Chem. Soc. Jpn. 1980, 53, 1832-1836.

(90) Robinson, R. A.; Stokes, J. M.; Stokes, R. H. Potassium hexafluorophosphate - an associated electrolyte. J. Phys. Chem. 1961, 65, 542-546.

(91) Uematsu, Y.; Araki, T. Electro-Osmotic Flow of Semidilute Polyelectrolyte Solutions. J. Chem. Phys. 2013, 139, 094901.

(92) Wiese, G. R.; Healy, T. W. Adsorption of Al(III) at the TiO2/H2O interface. J. Colloid Int. Sci. 1975, 51, 434-452.

(93) Bijsterbosch, B. H. The electrical double layer on silver iodide in the presence of organic molecules. Mededelingen van de Landbouwhogeschool Wageningen 1965, 65, 4, also Ph.D. Thesis, State University of Utrecht.

(94) Uematsu, Y.; Bonthuis, D. J.; Netz, R. R. Charged Surface-Active Impurities At Nanomolar Concentration Induce Jones-Ray Effect. J. Phys. Chem. Lett. 2018, 9, $189-193$. 
(95) Yates, D.; Healy, T. W. Titanium dioxide-electrolyte interface. Part 2. - Surface charge (titration) studies. J. Chem. Soc., Faraday Trans 1 1980, 76, 9-18.

(96) Lyklema, J. Electrical double layer on silver iodide. Influence of temperature and application to sol stability. Discus. Faraday Soc. 1966, 42, 81-90.

(97) Larson, I.; Attard, P. Surface Charge of Silver Iodide and Several Metal Oxides. Are All Surfaces Nernstian? J. Colloid Int. Sci. 2000, 227, 152-163.

(98) Overbeek, J. T. G. In Colloid Science; Kruyt, H. R., Ed.; Elsevier: Amsterdam, 1952; Vol. 1; p 115.

(99) Billett, D. F.; Hough, D. B.; Ottewill, R. H. Studies on the contact angle of the charged silver iodide-solution-vapour interface. J. Electroanal. Chem. 1976, 74, 107-120.

(100) Sakai, N.; Fujishima, A.; Watanabe, T.; Hashimoto, K. Quantitative Evaluation of the Photoinduced Hydrophilic Conversion Properties of TiO2 Thin Film Surfaces by the Reciprocal of Contact Angle. J. Phys. Chem. B 2003, 107, 1028-1035.

(101) Lyklema, H. J. Simple Hofmeister series. Chem. Phys. Lett. 2009, 467, 217-222.

(102) Kuznetsov, V.; Papastavrou, G. Ion Adsorption on Modified Electrodes as Determined by Direct Force Measurements under Potentiostatic Control. J. Phys. Chem. C 2014, 118, 2673-2685.

(103) Siria, A.; Poncharal, P.; Biance, A.-L.; Fulcrand, R.; Blase, X.; Purcell, S.; Bocquet, L. Giant osmotic energy conversion measured in a single transmembrane boron nitride nanotube. Nature 2013, 494, 455.

(104) Secchi, E.; Niguès, A.; Jubin, L.; Siria, A.; Bocquet, L. Scaling Behavior for Ionic Transport and its Fluctuations in Individual Carbon Nanotubes. Phys. Rev. Lett. 2016, 116, 154501. 
(105) Secchi, E.; Marbach, S.; Niguès, A.; Stein, D.; Siria, A.; Bocquet, L. Massive radiusdependent flow slippage in carbon nanotubes. Nature 2016, 537, 210. 


\section{Graphical TOC Entry}

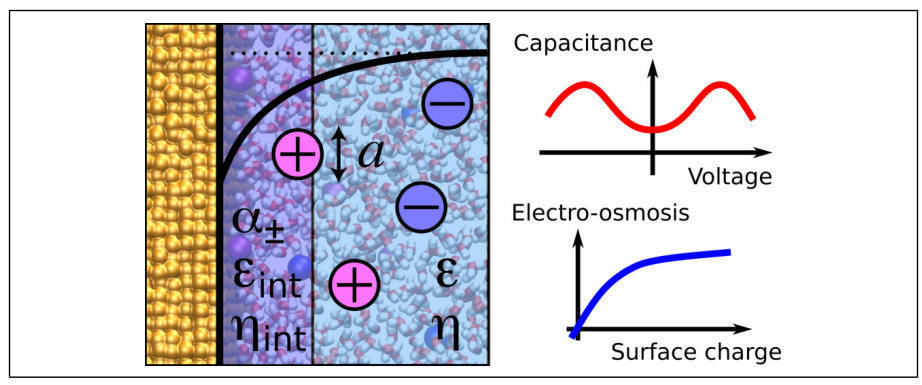

